\title{
Development of a Neutron List Mode Collar (LMCL) and a List Mode Response Matrix Analysis Concept
}

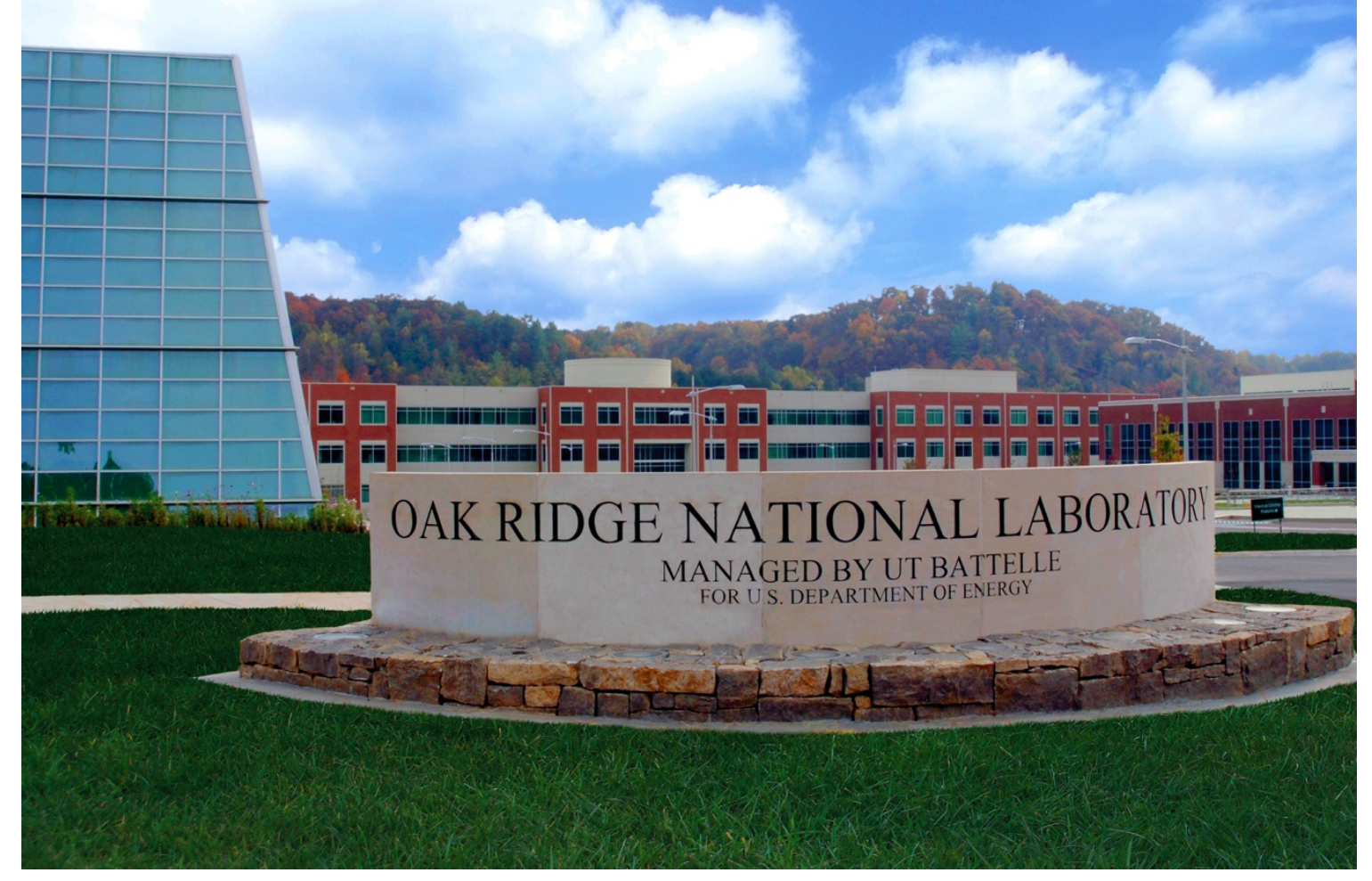

Louise G. Evans

Andrew D. Nicholson

Charles L. Britton Jr.

Kenneth J. Dayman

Milton N. Ericson

Angela S. Moore

Approved for public release.

June 2021

Distribution is unlimited. 


\section{DOCUMENT AVAILABILITY}

Reports produced after January 1, 1996, are generally available free via US Department of Energy (DOE) SciTech Connect.

Website www.osti.gov

Reports produced before January 1, 1996, may be purchased by members of the public from the following source:

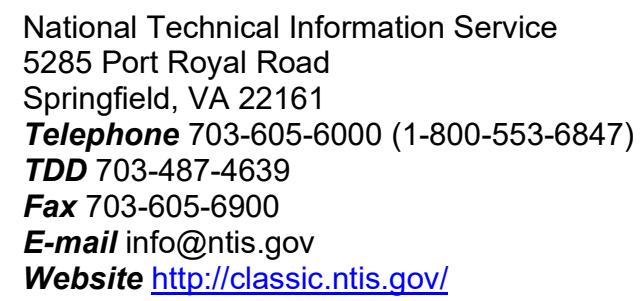

Reports are available to DOE employees, DOE contractors, Energy Technology Data Exchange representatives, and International Nuclear Information System representatives from the following source:

Office of Scientific and Technical Information

PO Box 62

Oak Ridge, TN 37831

Telephone 865-576-8401

Fax 865-576-5728

E-mail reports@osti.gov

Website https://www.osti.gov/

This report was prepared as an account of work sponsored by an agency of the United States Government. Neither the United States Government nor any agency thereof, nor any of their employees, makes any warranty, express or implied, or assumes any legal liability or responsibility for the accuracy, completeness, or usefulness of any information, apparatus, product, or process disclosed, or represents that its use would not infringe privately owned rights. Reference herein to any specific commercial product, process, or service by trade name, trademark, manufacturer, or otherwise, does not necessarily constitute or imply its endorsement, recommendation, or favoring by the United States Government or any agency thereof. The views and opinions of authors expressed herein do not necessarily state or reflect those of the United States Government or any agency thereof. 
Nuclear Nonproliferation Division

\title{
DEVELOPMENT OF A NEUTRON LIST MODE COLLAR (LMCL) AND A LIST MODE RESPONSE MATRIX ANALYSIS CONCEPT
}

\author{
Louise G. Evans \\ Andrew D. Nicholson \\ Charles L. Britton Jr. \\ Kenneth J. Dayman \\ Milton N. Ericson \\ Angela S. Moore
}

Date Published: June 2021

\author{
Prepared by \\ OAK RIDGE NATIONAL LABORATORY \\ Oak Ridge, TN 37831-6283 \\ managed by \\ UT-BATTELLE, LLC \\ for the \\ US DEPARTMENT OF ENERGY \\ under contract DE-AC05-00OR22725
}





\section{CONTENTS}

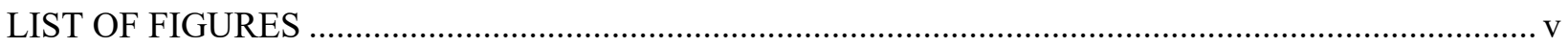

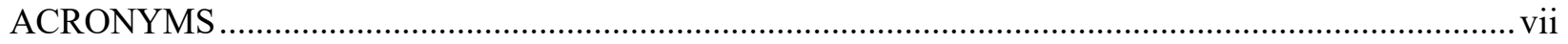

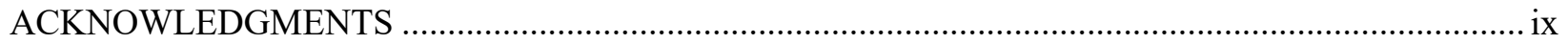

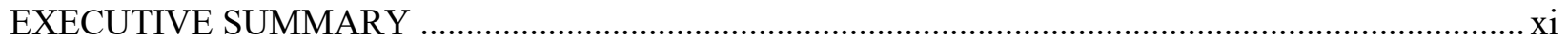

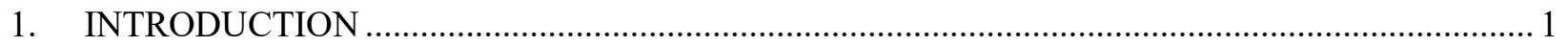

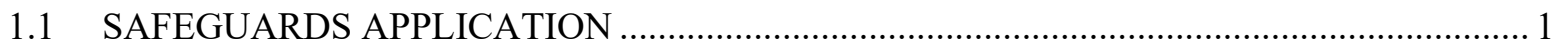

1.2 CURRENT TECHNOLOGY IMPLEMENTATION ...................................................... 1

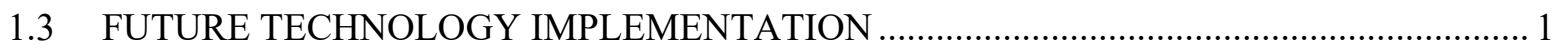

1.4 RESEARCH OBJECTIVES AND PROJECT GOALS …................................................. 2

1.5 BACKGROUND AND ADVANTAGES OF LIST MODE DATA ACQUISITION

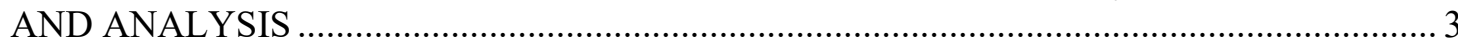

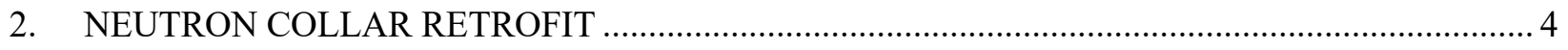

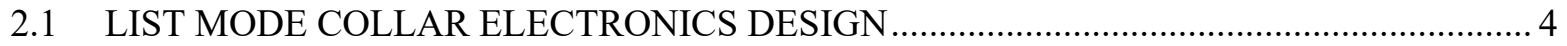

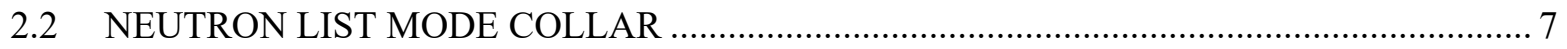

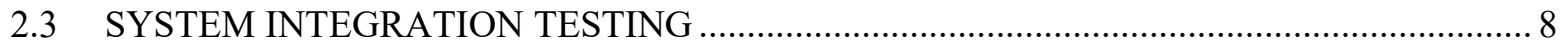

2.4 FINAL MEASUREMENT CAMPAIGN .............................................................................. 9

3. MODELING NEUTRON TRANSPORT IN THE LIST MODE COLLAR FOR ANALYSIS

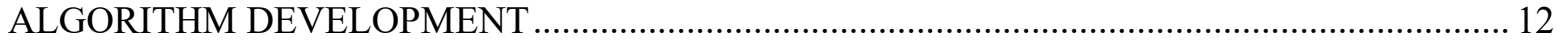

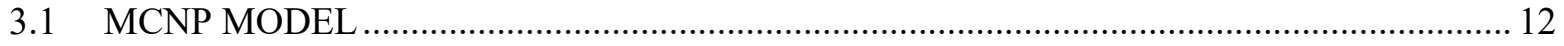

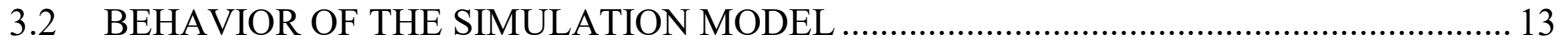

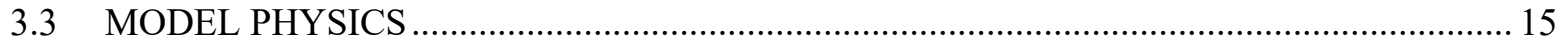

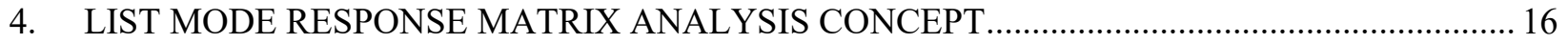

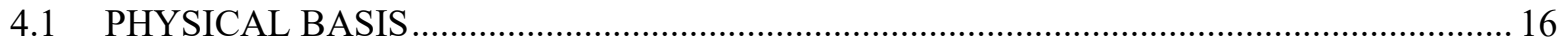

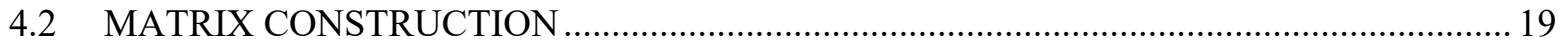

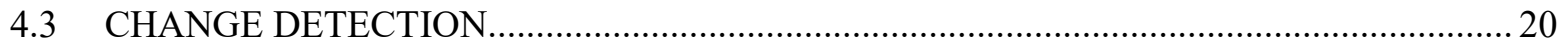

4.4 ALTERNATIVE APPROACH USING NEUTRON COINCIDENCE IMAGING.................23

4.5 PRELIMINARY STATISTICAL EVALUATION AND FEASIBILITY STUDY ................ 24

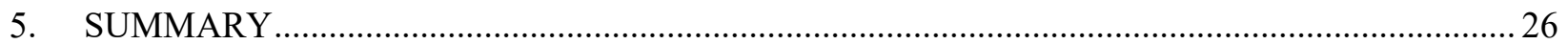

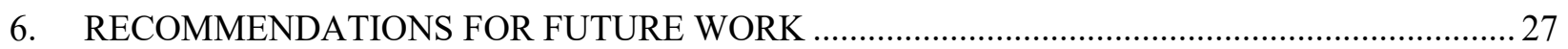

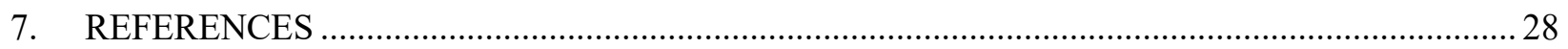





\section{LIST OF FIGURES}

Figure 1. (Left) PTR-32HV pulse train recorder list mode data acquisition system pictured alongside (right) the commonly used HH-MR shift register. .................................................... 3

Figure 2. Electronics configuration for original JCC-71 hardware..................................................... 5

Figure 3. (Left to right) Current preamplifier board; new preamplifier board (dimensions 1 in. $\times 2$

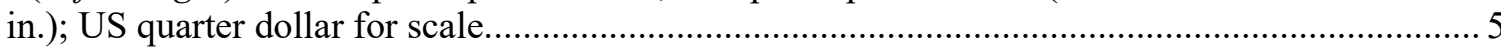

Figure 4. Electronics configuration for the new list mode collar............................................................. 6

Figure 5. (Left) JCC-71 detector bank configured with the new electronics to produce a sixchannel detector bank.

Figure 6. Full retrofit for the JCC-71 neutron coincidence collar to the ORNL LMCL.......................... 8

Figure 7. Measured doubles count rate (doubles per second) as a function of channel number (i.e., the measured doubles count rate response across all $18{ }^{3} \mathrm{He}$ detector channels for a ${ }^{252} \mathrm{Cf}$ source positioned at the center of the detector cavity for the LMCL [modified JCC-71]) [3].

Figure 8. Measured doubles count rate (doubles per second) as a function of channel number (i.e., the measured doubles count rate response across all $18{ }^{3} \mathrm{He}$ detector channels for a ${ }^{252} \mathrm{Cf}$ source positioned near detector ${ }^{3} \mathrm{He}$ tubes 6 and 7 for the LMCL [modified JCC-71]) [3] .......... 11

Figure 8. MCNP model of the UNCL detector encasing a fresh fuel assembly.

Figure 9. Simulated thermal mode doubles rate as a function of coincidence gate width for a 1,000 second count time.

Figure 10. Simulated thermal mode doubles rate relative uncertainty as a function of coincidence gate width for a 1,000 second count time.

Figure 11. 2D histogram of neutron interactions in each fuel cell plus the histogram of neutron detections in each ${ }^{3} \mathrm{He}$ detector. Detectors are labelled $1-18$.

Figure 12. Simulated $17 \times 17$ full fresh fuel assembly within the neutron collar detector (top).

Figure 13. 2D histogram of multiple detections for an AmLi interrogation source of strength $5 \times$ $10^{4}$ neutrons $\mathrm{s}^{-1}$ over 1 -hr measurement time in response to a full fresh fuel assembly.

Figure 14. (Top left) UNCL detector model.

Figure 15. 2D histogram of multiple neutron detections for a $5 \times 10^{4} \mathrm{n} / \mathrm{s} \mathrm{AmLi} \mathrm{source} \mathrm{over} \mathrm{a} \mathrm{1-hr}$ measurement time for a full assembly (Top Left) and 66 rods removed (Top Right), and the difference (Bottom Center) between full and partial defect scenario.

Figure 16. (Left) Pair efficiency matrix summed over all detectors for each fuel rod.

Figure 17. Calculated linear mass density for a full fuel assembly using a least squares algorithm on MCNP PTRAC calculated doubles rate and mass efficiency matrix.

Figure 18. Singles or total neutron counts as a function of detector number in response to a full fuel assembly and a group of eight pins removed in different locations throughout the fuel assembly...

Figure 19. Singles or total neutron counts as a function of detector number in response to a full fuel assembly and a group of eight pins removed in different locations throughout the fuel assembly. 



\section{ACRONYMS}

$\begin{array}{ll}\text { BWR } & \text { boiling water reactor } \\ \text { DOE } & \text { Department of Energy } \\ \text { DNN R\&D } & \text { Defense Nuclear Nonproliferation Research and Development } \\ \text { IAEA } & \text { International Atomic Energy Agency } \\ \text { LEU } & \text { low enriched uranium } \\ \text { LMCL } & \text { list mode collar } \\ \text { LVDS } & \text { low-voltage differential signal } \\ \text { MCNP } & \text { Monte Carlo N-Particle Transport } \\ \text { NDA } & \text { nondestructive assay } \\ \text { NNSA } & \text { National Nuclear Security Administration } \\ \text { ORNL } & \text { Oak Ridge National Laboratory } \\ \text { PTRAC } & \text { particle tracking } \\ \text { RVM-C } & \text { relevance vector machine classifier } \\ \text { TRL } & \text { Technology Readiness Level } \\ \text { UNCL } & \text { uranium neutron coincidence collar }\end{array}$





\section{ACKNOWLEDGMENTS}

The authors would like to acknowledge and thank the Safeguards Program of the US Department of Energy's National Nuclear Security Administration, Office of Defense Nuclear Nonproliferation Research and Development for supporting this work under project number OR16-List Mode for Collar-PD1La "List Mode Response Matrix for Advanced Correlated Neutron Analysis for Nuclear Safeguards." The authors would especially like to thank Program Manager Dr. Christopher Ramos for his technical guidance and vision. 



\section{EXECUTIVE SUMMARY}

This report was prepared for the Safeguards Program of the US Department of Energy's (DOE's) National Nuclear Security Administration (NNSA), Office of Defense Nuclear Nonproliferation Research and Development (DNN R\&D). The report presents the development of the neutron nondestructive assay system, the List Mode Collar (LMCL) for the project OR16-List Mode for Collar-PD1La "List Mode Response Matrix for Advanced Correlated Neutron Analysis for Nuclear Safeguards." The new list mode electronics developed under this project, and a spatial analysis concept called the List Mode Response Matrix are also described in this report. Analysis algorithms based on classification methods are published in a separate report [1].

This research addresses the need to expand the capabilities of current nondestructive assay systems used for nuclear safeguards applications and considers the sustainability of safeguards technologies by the development of a "retrofit" concept using electronics based on modern standards. Furthermore, employing list mode data acquisition enabled the development of a spatial analysis concept and empirical measurement of a spatial response not previously used for safeguards neutron counting applications or measured in a traditional neutron collar detector and, therefore, provides new capability.

\section{MISSION NEED AND SUSTAINABILITY}

As pressure on International Atomic Energy Agency (IAEA) safeguards resources continues to increase, there is a drive to essentially "do more with less" [2]. New ways of using current verification instrumentation and visualizing the collected data are needed to expand existing capabilities and thus extend the useful life of the current safeguards instrumentation fleet. This need for expanding current capabilities is also balanced by the need to maintain and sustain existing capabilities, as well as a need to retain simplicity of measurement system operations. This project completed a retrofit concept or prototype upgrade for current neutron counting systems, which although applied to a standard neutron collar, could in principle be applied to any safeguards neutron counting system with similar initial hardware. The upgrade includes the use of modern electronics to facilitate full list mode data acquisition; therefore, this research effort and technology development advances correlated neutron counting by facilitating new analysis capabilities for users, such as pattern recognition via spatial response measurements and will provide more diagnostic information on hardware performance. Also, the use of modern electronics standards improves the sustainability of those systems. Since no changes are made to the detectors themselves, only to the detector output signal processing chain, the electronics and hardware developed by this work could be fielded in systems currently in use (i.e., retrofit to existing neutron counting systems in the field). It is likely that existing systems can be upgraded with the new electronics and hardware developed by this project for a fraction of the cost needed for full system replacement. Furthermore, upgrading existing systems in this manner is a more cost-effective solution than replacing systems with other novel systems that might meet the same need. Additionally, it is expected that the technology developed in this project can be quickly fielded to help safeguard facilities.

\section{CHOSEN SAFEGUARDS APPLICATION}

Beyond the broader consideration of nondestructive assay system sustainability, improving the reliability of partial defect (rod removal) detection presents a specific technical need for safeguards. Therefore, the nondestructive assay of fresh low enriched uranium (LEU) fuel assemblies was chosen as the safeguards application for this research. The first thrust of this research investigated the retrofit of a standard neutron coincidence collar, and the second research thrust investigated list mode analysis algorithm development.

To address the technical needs in parallel with expanding the data acquisition capabilities of the neutron coincidence collar, a new advanced neutron correlation analysis method called the list mode response 
matrix was developed conceptually for future implementation in field-deployable software for fresh LEU fuel assay measurements. This method uses list mode data acquisition for every detector in a neutron coincidence collar to advance correlated neutron analysis by extracting a greater number of useful signatures from a neutron assay than are currently generated (e.g., spatial response). This method may enable the verification of spatial information about the assay item, such as the location of partial defects within a fresh fuel assembly, or the initial loading pattern of fuel and poison rods. The goal of the analysis component of this work is to enable nuclear safeguards inspectors to draw stronger conclusions about nuclear material diversion scenarios and partial defect identification for fresh fuel assemblies. This report describes the analysis concept, shows modeling and simulation results for fresh fuel source terms, and presents preliminary results of the sensitivity of the method to potential gross and partial defect scenarios.

\section{RETROFIT AND EXPANDED ANALYSIS CAPABILITY}

The prevalent neutron counting systems are composed of multiple ${ }^{3} \mathrm{He}$ detectors coupled to a series of power and signal processing electronics (i.e., power supply, preamplifier/amplifier/discriminator board, and derandomizer board housed in an electronics junction box), which in turn supplies neutron pulse train data to a shift register. The shift register processes the pulse data and, when combined with software, reports the total detected neutron counts and neutron coincidence rates. Currently, only a processed report of the combined raw data over all ${ }^{3} \mathrm{He}$ detectors, in terms of the neutron counting rate (i.e., singles and doubles), is reported from the shift register. In this work, the model JCC-71 Neutron Coincidence Collar detector was upgraded by retrofitting this standard uranium neutron collar detector with new electronics that enable list mode data acquisition. As a result, collected verification data can be analyzed in new ways. The list mode collar meets the data handling requirements of the original collar and provides backwards compatibility with traditional shift register electronics and coincidence analysis. Developed electronics enabled the viability and exploration of list mode analysis for spatial analysis of collected data. The ability to save the collected data and modify the analysis parameters offline provided improved analysis flexibility (e.g., changing the coincidence gate settings offline during the analysis eliminates the need to establish a fixed coincidence gate before measurement). Furthermore, this retrofit expands the data available for processing by enhancing the electronics used to supply time-stamped pulse data (also known as list mode) for each detector and for sets of detectors. To process this data, new software will ultimately need to be developed to give operators the same information as the original system, namely singles and doubles coincidence rates, but it can also provide new data streams such as spatial information. In this report, the overall concept of upgrading current neutron counting systems is discussed with respect to the potential increase in analysis capabilities provided by this research. A new neutron analysis method concept, the list mode response matrix, has been developed to analyze every pulse train from the detector array and thus enable the verification of spatial information about the assay item for safeguards inspections. Simulation results show spatial variation of the neutron count rates from fresh LEU fuel assemblies and indicate that this method could be used for change detection. Spatial response was also empirically demonstrated using the new collar and is summarized here and published in full in a separate journal article [3].

\section{END USERS}

The envisioned potential end users for this technology are domestic and international safeguards facilities employing neutron counting systems and organizations that oversee measurement campaigns, including the IAEA and DG-ENER Euratom. For some time, the IAEA has been interested in partial defect identification, or fuel pin diversion (i.e., type of partial defect). The system developed in this work could be useful to fulfill this need and recommendations for further development work needed to field the list mode collar system are also outlined. The modular nature of the developed electronics also facilitates their application to other neutron counting systems beyond just the neutron coincidence collar. For example, spatial response measurements could be performed in a neutron multiplicity counter retrofit with 
this electronics package for applications other than the safeguards verification of fresh nuclear fuel. The spatial response could also be used on new systems that already have list mode capability, that do not require the retrofit and would benefit from this new analysis. 


\section{INTRODUCTION}

\subsection{SAFEGUARDS APPLICATION}

International Atomic Energy Agency (IAEA) safeguards are applied to fuel fabrication plants as part of ensuring the peaceful use of nuclear fuel cycle technology. Nuclear materials accountancy is the primary method of IAEA safeguards applied at fuel fabrication plants. Nuclear material inside such a facility is in the form of uranium oxide fuel pellets within fuel rods within an assembly of those fuel rods (e.g., 264 fuel rods within a Westinghouse $17 \times 17$ fuel assembly design [4]). The final product, a fresh fuel assembly, is measured nondestructively during storage and before shipment (transfer to a reactor site) as part of the overall material balance verification at those facilities [5]. Measurements are performed on the fuel to verify operator nuclear material accountancy declarations of the ${ }^{235} \mathrm{U}$ enrichment and mass. The deployed safeguards technology for those measurements is the uranium neutron collar (UNCL) used to verify the ${ }^{235} \mathrm{U}$ mass per assembly. The neutron verification measurement determines linear mass density $\left(\mathrm{g}^{235} \mathrm{U} \mathrm{cm}^{-1}\right.$ ), which is combined with an active length gamma measurement $(\mathrm{cm})$ to determine the ${ }^{235} \mathrm{U}$ content of the entire assembly. The UNCL measures correlated neutrons from fission and the observable is the detected neutron coincidence rate, or doubles rate.

\subsection{CURRENT TECHNOLOGY IMPLEMENTATION}

In the current implementation of the UNCL, data acquisition is based on shift register electronics (e.g., the model JSR-15 multiplicity shift register [6]). The standard neutron coincidence analysis method uses only a single pulse train from 18 detector channels to determine the coincidence count rate used to reach safeguards conclusions. In this regard, the safeguards technology development community is facing a "catch-22" scenario regarding neutron assay: the need to simplify safeguards technology for inspector use and field deployment has meant that the potential of more nontraditional methods (namely, list mode) has not been fully utilized within existing systems, and new approaches to neutron counting have been constrained. Taking this example of existing neutron collar detectors, just one output signal (the sum of all detector channels) is made available to an inspector and input to a shift register. Consequently, just two measured signatures - the singles neutron rate and doubles neutron coincidence rate - are used as the basis for safeguards inspection measurements. Although the two signatures (coincidence) approach has historically been adequate for the determination of special nuclear material mass within a given fresh fuel assembly, the reliability of partial defect detection could be strengthened using a new assay mode by enabling a physics-based understanding of the origin of anomalies in the recorded neutron singles and doubles rates. Furthermore, partial defect identification could be strengthened for more complex fuel types including fresh mixed oxide fuel assemblies or fresh low enriched uranium (LEU) fuel assemblies containing burnable poisons.

\subsection{FUTURE TECHNOLOGY IMPLEMENTATION}

The technology solution lies within existing systems because more signatures can be obtained from a single assay with the straightforward extension to list mode or with minor modifications to the electronics junction box. A move toward list mode approaches for current safeguards neutron counting systems is likely to be more effective than the standard approach because it enables spatial information about the assay item to be obtained. This is achieved by using the spatial distribution of neutron counting rates within the detection system for analysis. Possible analysis approaches employ combinations of neutron counting rates (i.e., singles or correlations) from individual detectors (in list mode) or banks of detectors (e.g., front-to-back ratios or left-to-right ratios in neutron collars or slab counters, or ring ratios in neutron well counters) to provide more signatures from a given item assay. The future safeguards technology solution is envisioned to combine neutron list mode hardware with automated pattern recognition software in existing neutron collar detectors, to enable a spatial response-based analysis method to be 
used in a straightforward and transparent manner by an inspector. Neutron list mode enables a neutron counter to be used in an "imaging" mode by acquiring and processing digital pulses from multiple, individual detector channels. The new list mode response matrix method under development will advance conventional list mode analysis by displaying spatial information on the distribution of neutron counting rates within the detection system. For example, the singles hit pattern (i.e., pattern of neutron counts across individual channels) or local coincidence matrix (i.e., cross-correlations in adjacent detector channels) are viable signature candidates for this method. Each measured fuel assembly would, therefore, have its own recognizable pattern, and deviations in this pattern (outside statistical fluctuations) could be recognized by software. Recognizable patterns of multiple measured signatures are difficult to replicate and harder to spoof; therefore, this approach increases the reliability of partial defect detection for an inspector. A list mode approach is also useful for heterogeneous items where nuclear material is distributed within complex item matrices (e.g., heterogeneous blanket/fuel pin assemblies, waste assay). Furthermore, this approach has several general benefits for detector calibration and maintenance, which are discussed in Section 1.5. These benefits are reviewed in [7], where select list mode calibration features are also demonstrated empirically.

This discussion leads to the project research question: Can imaging and pattern recognition be performed in the existing UNCL to facilitate change detection with the upgrade to list mode?

\subsection{RESEARCH OBJECTIVES AND PROJECT GOALS}

The overall research concept is to leverage developments in other fields to enhance current safeguards technologies and strengthen safeguards. Ultimately, this will be achieved by utilizing other general technology trends including smaller components and greater portability; improved data storage; and modern electronics.

The research objectives for this work were to

1. develop an advanced correlated neutron analysis method called the list mode response matrix,

2. demonstrate a prototype "retrofit package" to be developed and ultimately provided to the IAEA and the domestic safeguards community; and

3. provide a list mode calibration procedure.

Meeting these objectives increased the system Technology Readiness Level (TRL) toward achieving the following project goals:

1. Extend correlated neutron analysis by extracting more useful signatures from a neutron assay of fresh fuel than are currently generated (The signature of interest is spatial information to enable improved partial defect detection (rod removal) and attain high confidence in partial defect detection.)

2. Strengthen safeguards by increasing the deterrent

3. Ensure that the new list mode collar (LMCL) is more robust than the current UNCL by design

4. Retain the overall system ease of use and not alter the current inspection method or concept of operations

This report describes the development of the LMCL and list mode response matrix method, which meet the first and second research objectives. 


\subsection{BACKGROUND AND ADVANTAGES OF LIST MODE DATA ACQUISITION AND ANALYSIS}

List mode data acquisition provides a complete record of a given measurement, and offline analysis is possible. A list mode data file provides the arrival time and counting channel of every detected neutron event. The PTR-32 list mode data acquisition module pictured in Figure 1 has recently been approved for inspection use by the IAEA, which aids the deployment of list mode applications. However, the current implementation of neutron coincidence collar technology uses data acquisition based on shift register electronics. Standard coincidence analysis uses only a single pulse train from the sum of 18 detector channels to determine the neutron coincidence count rate used to draw safeguards conclusions. Therefore, list mode analysis is not being fully utilized in current neutron nondestructive assay (NDA) systems. One historical reason for this is that current neutron collar technology does not support full list mode data acquisition because the technology is not equipped with the necessary hardware to take advantage of this approach. List mode analysis is also made possible with modern computers and data storage, which were not available when the original neutron collar detectors were designed, optimized, and fielded. Moving toward list mode data acquisition and analysis fully enables inspection instruments to leverage the new PTR-32 capability. There is a corresponding driver to develop inspection procedures and calibration methods for this safeguards application area that employ list mode analysis.

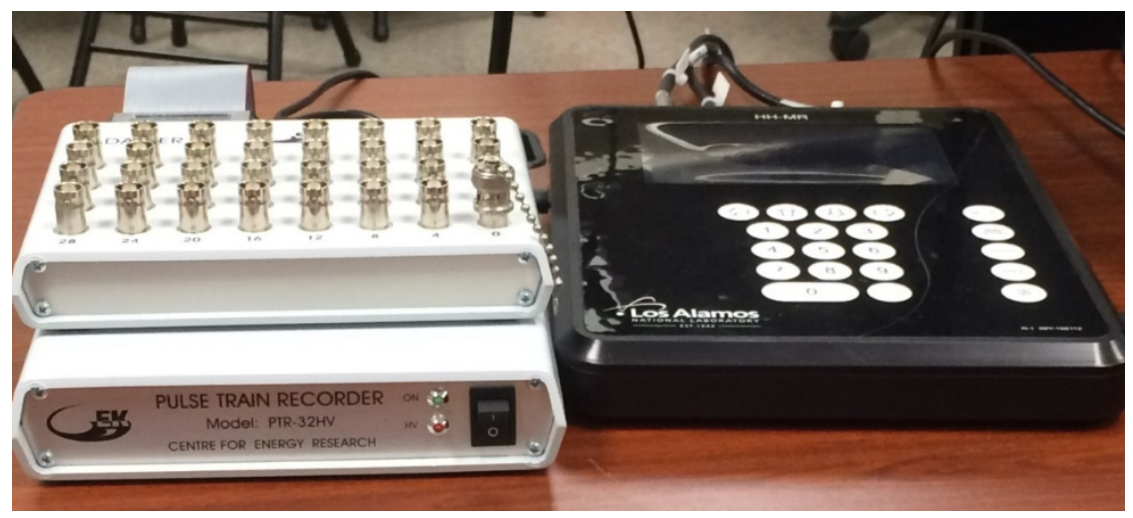

Figure 1. (Left) PTR-32HV pulse train recorder list mode data acquisition system pictured alongside (right) the commonly used HH-MR shift register.

In terms of its advantages, the list mode data file can be replayed offline for further analysis, and multiple analysis options can be employed. Therefore, one benefit of retaining the list mode file is to assist with reach back activities because storage of greater information in the list mode file provides more information at the time of expert review at the IAEA in Vienna than the current IAEA Neutron Coincidence Counting software output file, which provides only the histograms and the derived neutron counting rates from the detected pulse train from shift register data acquisition. Therefore, list mode could assist with and enhance expert data review. Adopting a list mode approach for future inspections also has the potential to lead to the creation of a database containing this information, which would support comparisons of measurements of similar items with historical measurements using advanced algorithms. This could be used for data review activities at the IAEA in Vienna, or for field deployment, which would allow inspectors to see if something changed over a period of successive measurements for a single item and raise questions if an item under assay differs substantially from many other similar items. Spoofing 18 channels of data is more difficult than 1 channel of data.

List mode provides faster and easier characterization. It enables observation of the entire time behavior of the detection system and, therefore, provides an enhanced physics-based understanding of the system characteristics. This means that a greater number of signal parameters can be extracted at the calibration 
stage. For example, the Rossi-alpha distribution (distribution of the time between events), which was not possible with shift register data acquisition. Traditional time gating structure can be overlaid on the pulse train, but with many different gates and list mode is not constrained to one fixed predelay and gate. This increases the available analysis options (e.g., die-away time derived from offline analysis by applying multiple coincidence gates to the stored pulse train) and potential to obtain greater information from a given assay. List mode also provides a significant diagnostic benefit and greater information on the stateof-health of instruments operating in the field and each individual detector channel. For example, individual channel behavior can be observed and if a preamplifier fails or a detector breaks, and this can be accounted for in offline analysis to provide enhanced self-diagnostics of the instrument. Issues such as double pulsing can also be quickly identified and diagnosed. In this regard, list mode enhances measurement control and quality assurance. List mode also provides greater redundancy. For example, it assists with preamplifier failure, detector leakage, and bank failure.

\section{NEUTRON COLLAR RETROFIT}

\subsection{LIST MODE COLLAR ELECTRONICS DESIGN}

This section of the report documents the list mode collar electronics design and the integrated system. The details of the list mode collar electronics and overall system design were previously reported at the IAEA Safeguards Symposium, November 2018 [8] and are included below with additional discussion. The integrated system was not previously reported and is new to this report.

The new LMCL is based on the original JCC-71 neutron coincidence collar [9]. As described in the current implementation of correlated neutron counting (both coincidence and multiplicity), data acquisition uses shift register electronics (e.g., the model JSR-15 multiplicity shift register). List mode approaches are gradually being considered and adopted for IAEA safeguards applications, and the PTR32 list mode data acquisition unit is equipment approved for IAEA use. However, changes to the hardware of existing neutron counters are needed to take full advantage of the potential of list mode data acquisition, which necessitates this developmental work. Traditional neutron counting electronics for IAEA NDA neutron counting systems employ preamplifier-shaper networks that output transistortransistor logic (TTL) pulses in response to a detected neutron event. A typical counting channel comprises several ${ }^{3} \mathrm{He}$ gas-filled proportional counters that are grouped together and serviced by a single preamplifier. The logical outputs of all the amplifiers are summed to create a single pulse train for the system. The current hardware configuration of the JCC-71 neutron coincidence collar (or UNCL) has six detectors (gas-filled ${ }^{3} \mathrm{He}$ proportional counters) per preamplifier, as illustrated in Figure 1. Therefore, list mode data acquisition from all 18 channels (active mode) or 24 channels (passive mode) is not possible with the current hardware configuration. 


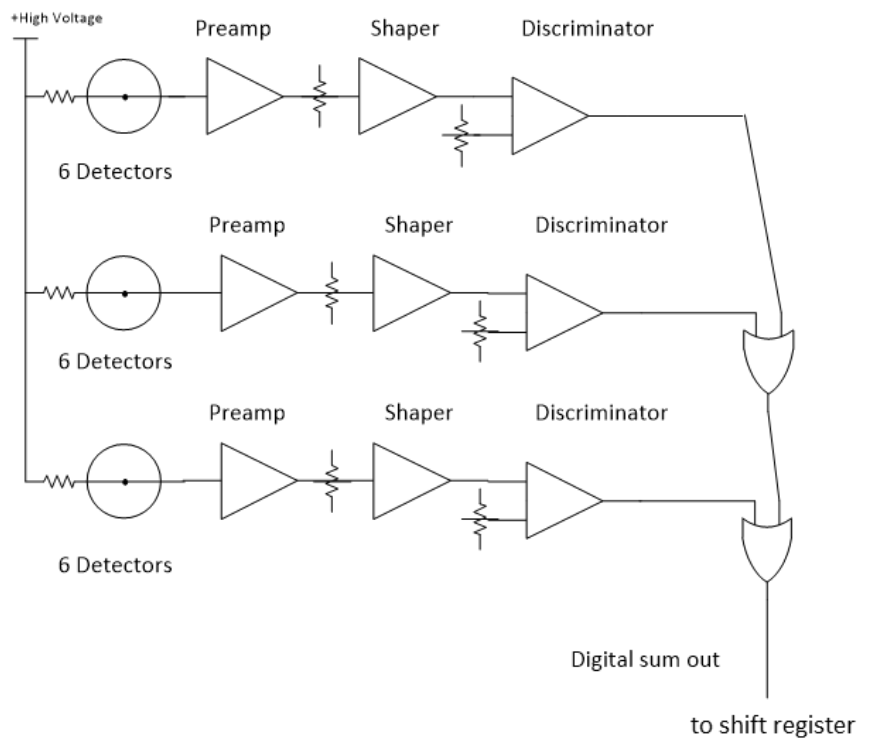

Figure 2. Electronics configuration for original JCC-71 hardware.

The model JCC-71 neutron coincidence collar detector was retrofit with new electronics and one chargeamplifier-discriminator-line drive per detector to provide multichannel list mode data acquisition (24 channels for the passive mode; 18 channels for the active mode). The design concept of the new neutron counting electronics is to be able to fit them inside the existing collar electronics junction box, so that the detection system itself, as well as the overall size and footprint of the collar, remains unchanged. The prototype retrofit package developed for this project includes a list mode calibration procedure for use with the PTR-32 and backwards compatibility with the JSR-15.

New electronics were designed and assembled at Oak Ridge National Laboratory (ORNL). Steps included completing the technical specification, design, development, prototype fabrication and testing, parts production, assembly, and final testing of the new preamplifier and 6-channel motherboard. The new preamplifier is based on a printed circuit board design and is shown in Figure 3 (left). The new architecture has six independent channels, and list mode data are available for each detector channel of each bank (or slab). This new layout is illustrated in Figure 3 (right), which shows the six preamplifiers mounted on a motherboard that distributes the $5 \mathrm{~V}$ DC power and the high voltage bias to each detector.
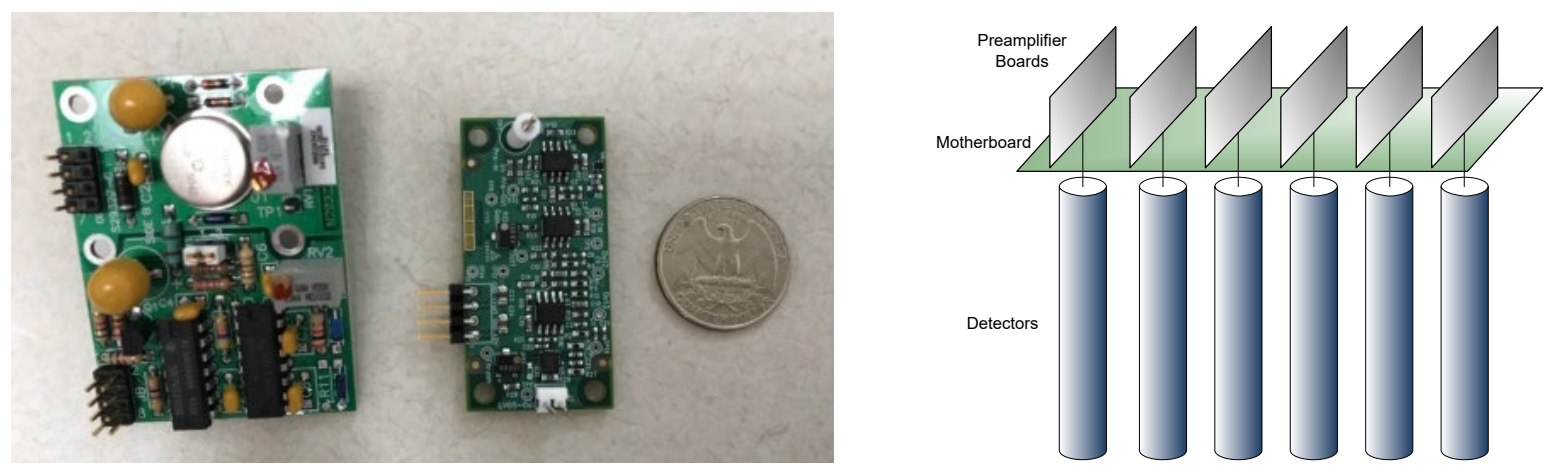

Figure 3. (Left to right) Current preamplifier board; new preamplifier board (dimensions 1 in. $\times 2$ in.); US quarter dollar for scale. Note, the functionality of these two boards is not strictly equivalent, and the new board connected to the motherboard achieves the same functionality. (Far right) Basic architecture of the electronics for a single detector bank. 
Figure 4 illustrates the new electronics design. Each detector has its own individual signal processing chain to tag the detected neutron events in time and detector channel. The new approach is to place a preamplifier and discriminator on each detector with its own set of signal lines going into a digital processing unit. The digital processing for this phase of the electronics takes the time-over-threshold signal from each discriminator and converts it to a fixed $50 \mathrm{~ns}$ width TTL pulse. The output signals are then combined for use with a traditional shift register. This approach provides the sum of all channels for backwards compatibility, as well as the sum of each bank (or slab) and the individual 18 or 24 channel outputs.

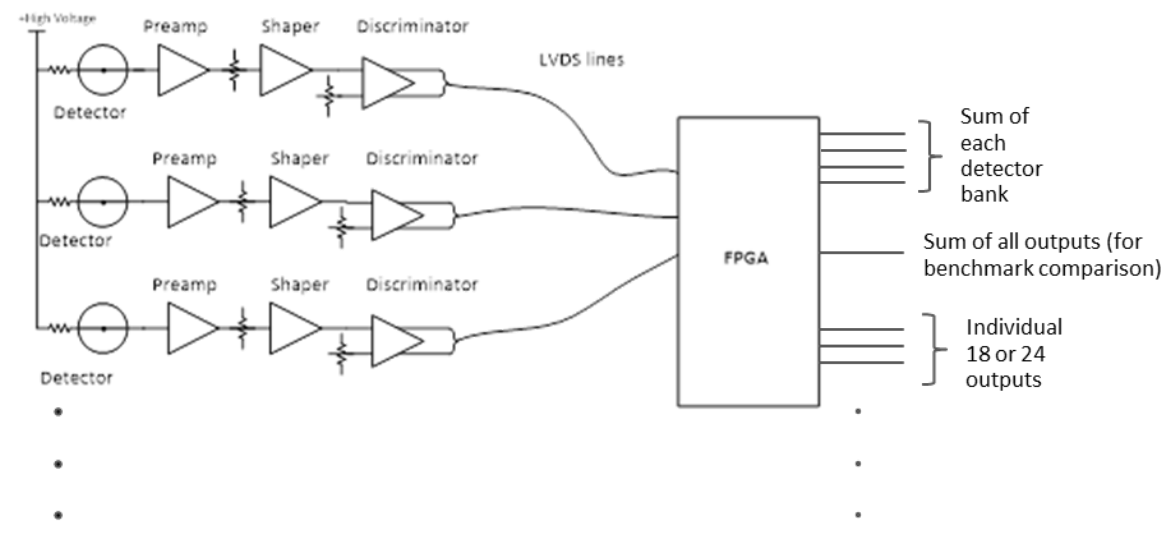

Figure 4. Electronics configuration for the new list mode collar.

The neutron counting electronics were constructed to improve both the capability and sustainability of existing neutron counting systems. The new electronics design uses the latest generation, low-voltage, commercial operational amplifiers, which are based on modern standards. Signals are output from the discriminators as low-voltage differential signal (LVDS) standard logic output instead of a TTL output signal traditionally used in IAEA neutron counting applications. LVDS output was chosen as a modern standard for the reasons outlined below. Consequently, an external printed circuit board was also designed and built, the "ORNL neutron collar processor," to convert the LVDS output from the discriminators to TTL pulses. All 4 detector banks ( 24 channels) were retrofit with the new preamplifiers and motherboard. It was possible to recycle the existing junction box housing and fit the new electronics within that box. The only modification was to 3D print a new lid for each junction box with a small notch for the new cable to pass out of the junction box to the converter board. Figure 5 shows these design elements with the complete assembly on the left and the ORNL neutron collar processor board on the right. 

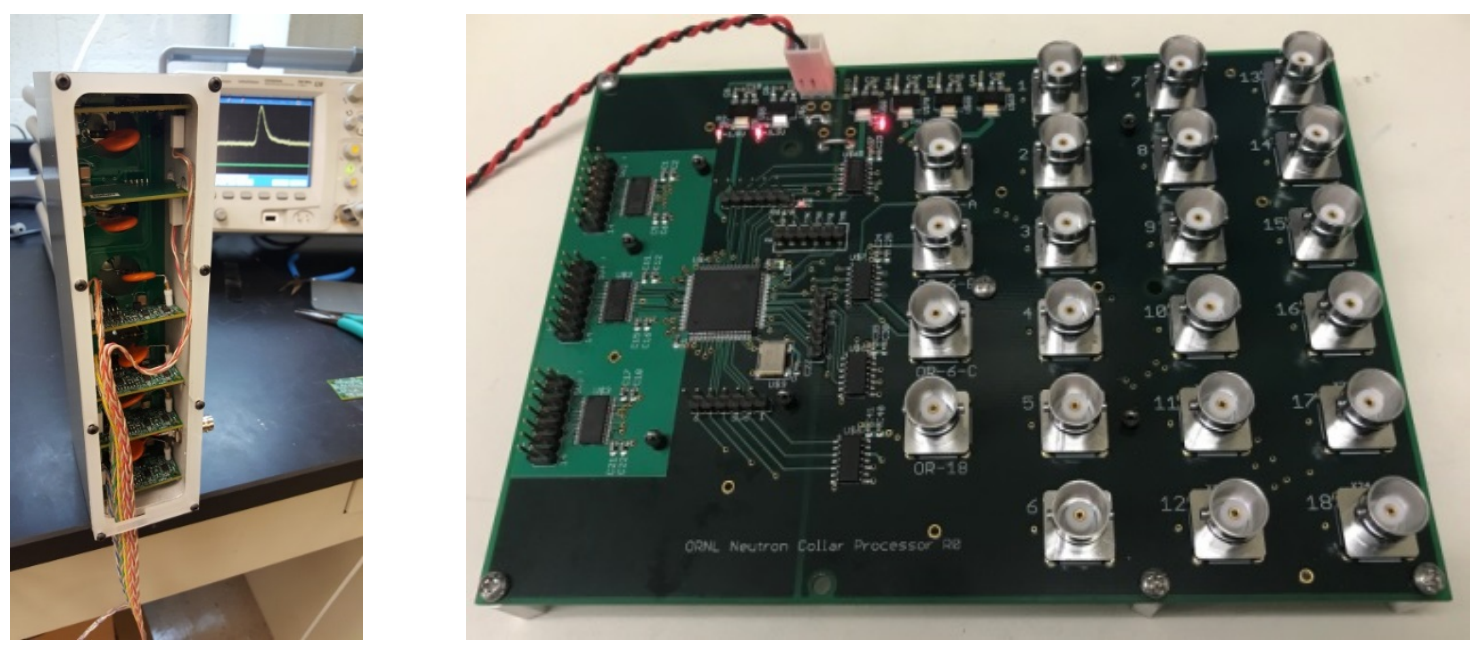

Figure 5. (Left) JCC-71 detector bank configured with the new electronics to produce a six-channel detector bank. (Right) The ORNL neutron collar processor.

The modular and scalable nature of the new electronics design means that it could be applied to other neutron counting systems. LVDS output was chosen as a modern standard to minimize the influence of future component obsolescence and therefore to help sustain those systems. Other reasons for this choice of output are listed:

1. Reduced signal injection-Single ended TTL signals are known for feeding fast edges back into the originating circuits and into other colocated circuits thus causing false triggers. LVDS is a fully balanced signal that has a low voltage swing and is inherently noninterfering.

2. Uses twisted-pair cables - LVDS used standard $100 \Omega$ twisted pair cables, which are small, inexpensive, and can be run without shielding.

3. LVDS is a standard interface - LVDS is a standard interface on all modern field-programmable gate arrays, whereas TTL requires external logic and level shifters to work with new electronics.

4. Future compatibility-It is anticipated that future data acquisition systems will accept LVDS directly.

Commercial-off-the-shelf components were used for the preamplifier board. This type of retrofit also demonstrates that upgrading existing systems with new hardware (and ultimately, software) is more costeffective than system replacement. Replacing individual components, rather than the full system, maintains the NDA system reliability and ease of use.

\subsection{NEUTRON LIST MODE COLLAR}

The retrofit of the full neutron collar detector (four detector banks) was completed, along with final testing of the electronics. Figure 6 shows the LMCL upgrade of the JCC-71 neutron coincidence collar. 


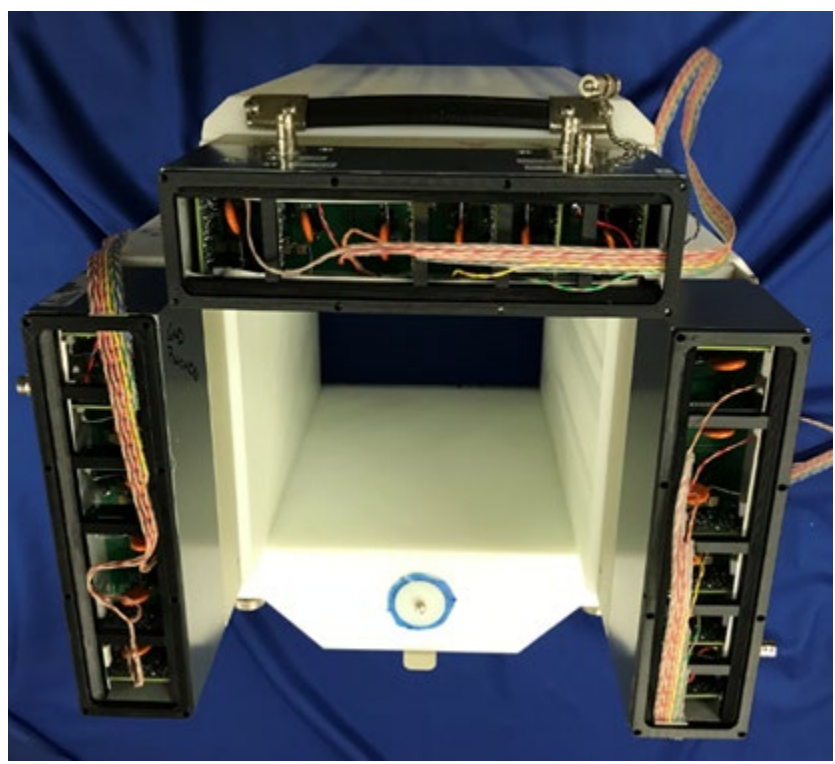

Figure 6. Full retrofit for the JCC-71 neutron coincidence collar to the ORNL LMCL.

The following improvements were made to the collar preamplifier motherboard revision 1 (R1), compared with the original $\mathrm{R} 0$, before final integration.

- Added local voltage regulators for each preamplifier supply voltage

- Routed HV signals for improved isolation

- Decreased the hole size in the motherboard for connection to the ${ }^{3} \mathrm{He}$ tube, which allowed the HV components to be placed further from the motherboard edge/housing (improved voltage standoff)

\subsection{SYSTEM INTEGRATION TESTING}

The tested collar detector banks were connected to the pulse processor board, and testing commenced as an integrated system. The configuration for this final test setup used motherboards in 3 banks of $18{ }^{3} \mathrm{He}$ tubes with 18 ORNL preamplifiers, producing an LVDS that was processed by the shielded converter board within the external box. During these tests, the system was not functioning as expected. During December 2018, an electrical event (HV discharge event) led to the failure of three preamplifier boards and damaged one of the six-channel modules. Steps were taken to understand the exact cause of the event, correct for this, and complete the experimental performance testing.

After the HV discharge event, the 3 six-channel preamplifier modules (minus the spare six-channel module previously damaged) were modified to better prevent future HV discharge events. This second round of modifications included:

- The LVDS signal pair (the full ribbon cable has six twisted pairs) from each of the six preamplifiers was mechanically fixed to the inside of the grounded enclosure with polyimide tape to prevent potential contact with the HV coupling capacitor of each channel.

- Teflon separators were added between the input power BNC of the unit and the adjacent HV preamplifier coupling capacitor.

$\circ$ The power cable ( $+6 \mathrm{~V}$, ground) to the motherboard passes near one of the HV capacitors. Although there is no evidence of this being a failure point, improving the standoff using a Teflon separator was advised and added. 
- A Teflon sheet was placed on top of the six-channel unit preamplifier cavity (below the lid and 3Dprinted spacer) to prevent the LVDS signal cables located near the lid from sagging into the space where the HV coupling capacitors are located.

- It was verified that all preamplifier housing lids were firmly attached with screws. Longer screws were needed for lid closure. The purpose of the installation of the new screws was to affix the lid and ensure that the lid was grounded to the case.

- The preamplifier thresholds were adjusted to match the first test measurement campaign using the single preamplifier bank.

\subsection{FINAL MEASUREMENT CAMPAIGN}

The final measurement campaign was performed using the prototype LMCL (or modified JCC-71). The configuration for the final measurement campaign was the motherboards in 3 detector banks, $18{ }^{3} \mathrm{He}$ tubes with 18 ORNL preamplifiers, producing an LVDS that was processed by the shielded converter board within the external box. Each individual channel is labeled, and the associated neutron pulse trains are processed separately offline to derive the doubles rate per channel. This configuration mimics the active system configuration, except an $\mathrm{AmLi}(\alpha, n)$ interrogation source was not present. Instead, a ${ }^{252} \mathrm{Cf}$ spontaneous fission neutron source was measured, which provided a source of coincident neutrons for testing the LMCL. The measurement campaign was performed to demonstrate the spatial response that could be achieved using all 18 detector channels. The final spatial measurement results were published in a journal article [3] alongside a comparison of a LMCL detector bank with an original JCC-71 detector bank.

Selected preliminary results are provided here. The spatial response function of the LMCL is shown in Figure 7 below for a ${ }^{252} \mathrm{Cf}$ source positioned at the center of the collar detector cavity. The source was centered in both the radial (i.e., equidistant from the edge of the high-density polyethylene blocks of the three LMCL detector banks and source moderator block) and axial directions. The LMCL spatial response is expressed as the measured doubles count rate as a function of detector channel number for all 18 detector channels. With the ${ }^{252} \mathrm{Cf}$ source in the central position, the detected doubles rates are higher in the detectors near the source. The detectors in the corners and furthest away from the ${ }^{252} \mathrm{Cf}$ source (detectors 6,7,12, and 13) have the lowest doubles rates. This is due to corners of the collar that do not contain polyethylene, which leads to a higher probability of neutron escape. Therefore, the number of neutrons thermalized and captured by the detectors near the corners is reduced relative to other detector locations. The spatial response in Figure 7 can be compared to the off-center measurement shown in Figure 8. Figure 8 shows the spatial response for relocating the same ${ }^{252} \mathrm{Cf}$ source to the corner of the detector cavity near detectors 6 and 7. Figure 8 shows that the measured doubles rates are significantly higher in detectors 6 and 7 than the doubles rates in those detectors from measuring the source at the center (Figure 7). This difference in the doubles count rates shows the change in source location. With the source positioned in the corner, the doubles count rates in tubes 4-9 are greater than those measured with the source in the center.

This experiment demonstrates that measurable differences in neutron doubles count rates can be observed depending on the position of the ${ }^{252} \mathrm{Cf}$ source within the LMCL. Therefore, a spatial response function can be measured using a neutron collar detector retrofit with list mode electronics. 


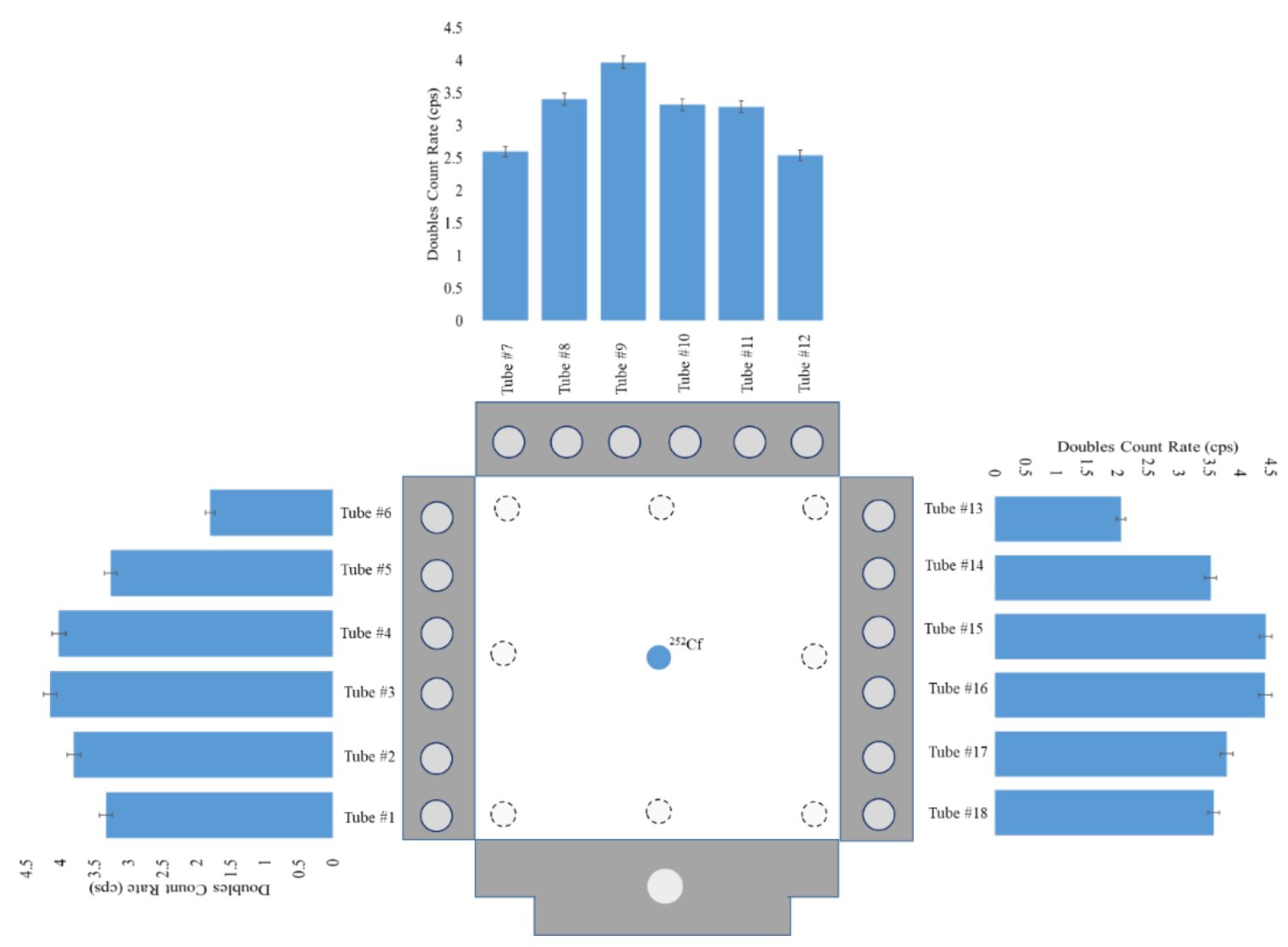

5

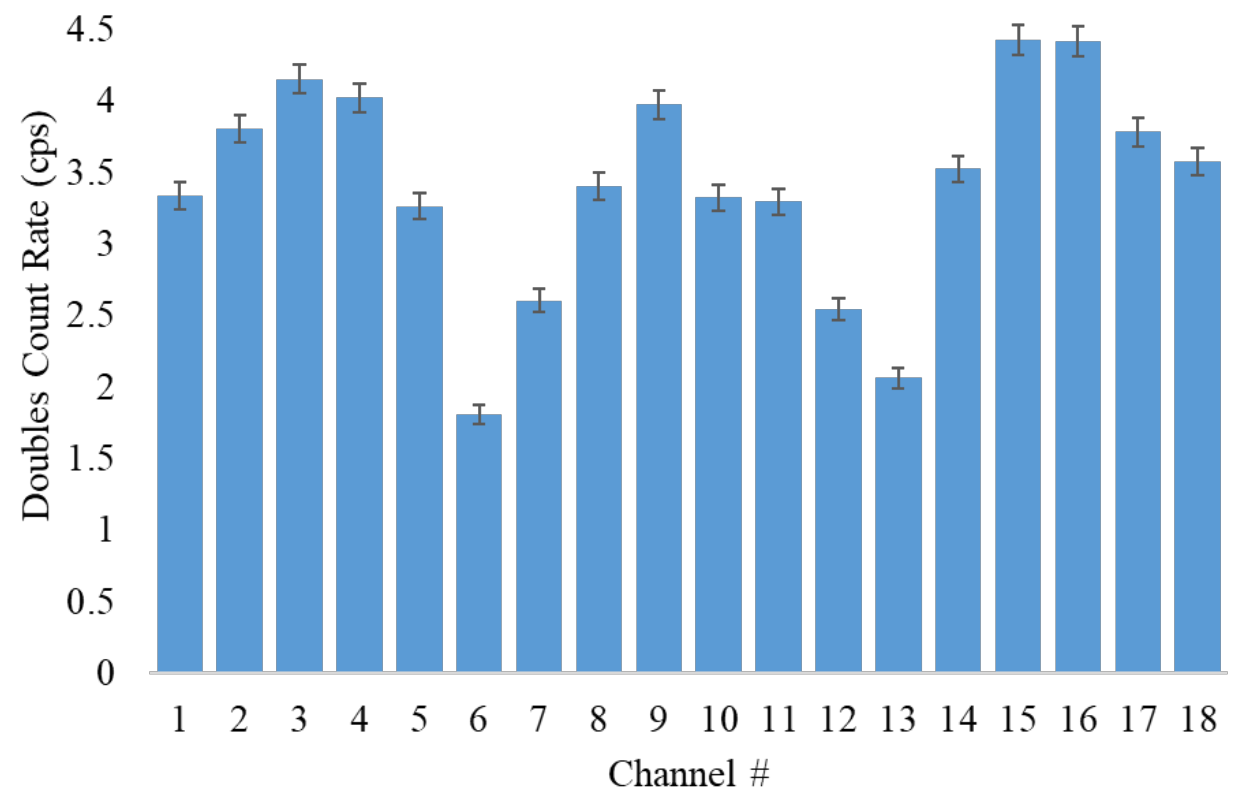

Figure 7. Measured doubles count rate (doubles per second) as a function of channel number (i.e., the measured doubles count rate response across all $18{ }^{3} \mathrm{He}$ detector channels for a ${ }^{252} \mathrm{Cf}$ source positioned at the center of the detector cavity for the LMCL [modified JCC-71]) [3]. 

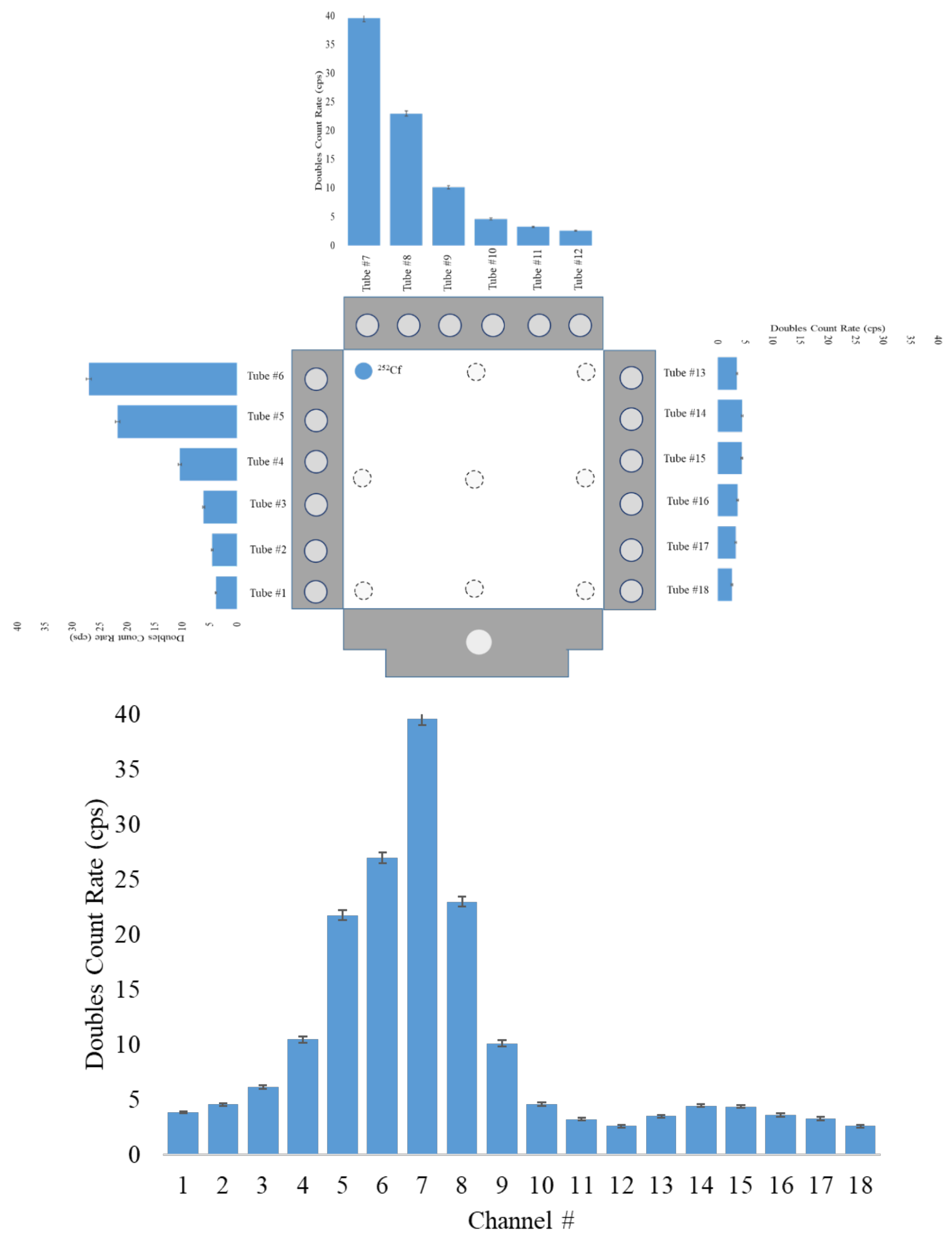

Figure 8. Measured doubles count rate (doubles per second) as a function of channel number (i.e., the measured doubles count rate response across all $18{ }^{3} \mathrm{He}$ detector channels for a ${ }^{252} \mathrm{Cf}$ source positioned near detector ${ }^{3} \mathrm{He}$ tubes 6 and 7 for the LMCL [modified JCC-71]) [3]. 


\section{MODELING NEUTRON TRANSPORT IN THE LIST MODE COLLAR FOR ANALYSIS ALGORITHM DEVELOPMENT}

The first step in the feasibility study of an analysis method based on spatial response was to set up neutron transport models for fresh fuel assemblies within a neutron collar detector array. The second step was to build a simulation of the response matrix and determine the potential capability of the method for change detection. The final step, which is a subject for future work, is to perform a detailed statistical analysis as a function of several assay scenarios including a detailed statistical analysis as a function of the assay time available to an inspector.

Modeling and simulation were the chosen methods for the development of the list mode response matrix method and subsequent classification methods because it is not possible to access fresh fuel assemblies covering a diverse range of nuclear designs. Simulation enables optimization of the analysis method to be performed over a diverse range of parameter space including the following.

- Fuel configuration (nuclear design)

○ Enrichment (representative range: $3.5-4.95 \mathrm{wt} \%{ }^{235} \mathrm{U}$ )

$\circ$ Gadolinium loadings (representative range: $8-24$ rods)

$\circ \quad$ Size of pin array $(17 \times 17$ or other designs)

- Count time or assay time

- Number of detectors included in the matrix

- Sum of all channels (current implementation, UNCL)

- Individual channels (future direction, LMCL)

- Individual detector banks (possible with current implementation, UNCL, but not used)

- Channel groupings (future direction, LMCL)

- Cross-correlations across channels (future direction, LMCL)

A full parametric study is necessary but must be built from the base case. The starting point of the simulations was a $17 \times 17$ light water reactor fresh fuel assembly of the Westinghouse type of design [4].

\subsection{MCNP MODEL}

The starting point for the model was the Los Alamos National Laboratory Monte Carlo N-Particle Transport (MCNP) [10] model of the UNCL. This was obtained by request from NA-241 Safeguards Technology Development because that model was used for the NA-241 Neutron Rodeo project [11], which was a round-robin intercomparison of the performance of a range of ${ }^{3} \mathrm{He}$ free detector technologies for neutron collar detector measurements. These ${ }^{3} \mathrm{He}$ free technologies were modeled in the same geometry as the UNCL and compared against the Los Alamos National Laboratory UNCL model.

The model was peer reviewed, and several modifications were made, including changing the density of the polyethylene moderator and making the fresh fuel source term more realistic by updating the density of the fuel. Figure 8 shows the MCNP model geometry. The model of the UNCL detector is shown encasing a fresh fuel assembly. 


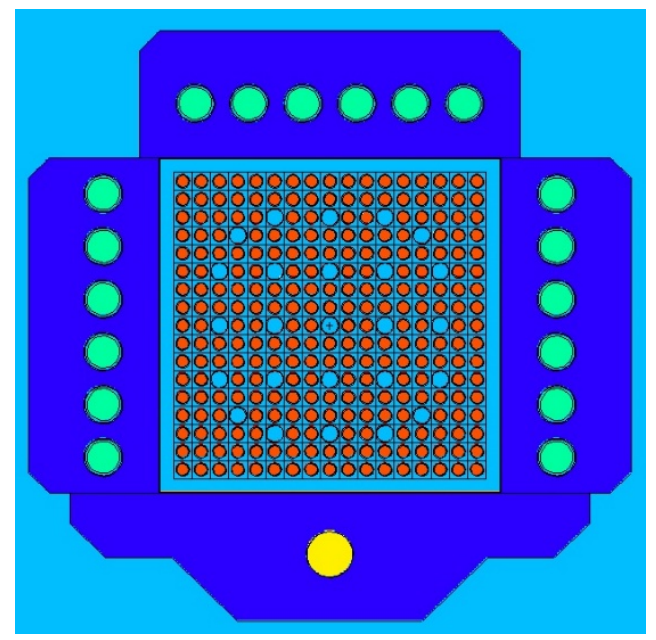

Figure 8. MCNP model of the UNCL detector encasing a fresh fuel assembly. The ${ }^{241} \mathrm{AmLi}$ source is shown in yellow; the high-density polyethylene moderator is dark blue; the ${ }^{3} \mathrm{He}$ tubes are green; the fuel assembly is red; and the surrounding air is light blue.

A neutron collar detector measurement of a $17 \times 17$ pin array pressurized water reactor uranium fresh fuel assembly was chosen as the basis for the development of this new analysis method because it represents one of the more straightforward NDA systems and measurement scenarios. In the future, this analysis method may be applicable to the assay of other fresh fuel designs and fuel compositions (e.g., mixed oxide and thorium) or extended to the assay of spent nuclear fuel.

\subsection{BEHAVIOR OF THE SIMULATION MODEL}

The MCNP tally results were post-processed with realistic count times and representative source strengths. Table 1 provides initial results on the magnitudes and relative uncertainties of the neutron counting rates that can be expected using this system.

\begin{tabular}{lccccc}
\hline \multicolumn{1}{c}{ Mode } & $\begin{array}{c}\text { Count time } \\
\text { (s) }\end{array}$ & $\begin{array}{c}\text { Singles } \\
\text { (cps) }\end{array}$ & $\begin{array}{c}\text { Singles rel. unc. } \\
\mathbf{( \% )}\end{array}$ & $\begin{array}{c}\text { Doubles } \\
\text { (cps) }\end{array}$ & $\begin{array}{c}\text { Doubles rel. } \\
\text { unc. } \\
\text { (\%) }\end{array}$ \\
\hline No-Cd (Thermal) & 1,000 & $2,948.76$ & 0.06 & 172.31 & 1.76 \\
No-Cd (Thermal) & 3,600 & $2,948.76$ & 0.03 & 172.31 & 0.53 \\
Cd (Fast) & 1,000 & $1,015.14$ & 0.10 & 12.54 & 6.64 \\
Cd (Fast) & 3,600 & $1,015.14$ & 0.05 & 12.54 & 2.06 \\
\hline
\end{tabular}

Using the simulated rates, two typical calibration experiments were replicated using another simulation, which is indicative of the model behaving as expected. These plots confirm the physics behavior of the model and are purely illustrative, rather than serving as a formal benchmark. Figure 9 shows the simulated thermal (no $\mathrm{Cd}$ ) doubles neutron counting rate as a function of coincidence gate width for a 1,000 second counting time, which is simulating the action of a shift register. As the coincidence gate width increases, the number of real coincidence pairs also increases. This is because a greater number of neutron coincidences are sampled from the neutron pulse train. However, the plot begins to reach a plateau as the maximum number of real coincidences (i.e., detected pairs of induced fission neutrons coincident within the gate width) is reached. 


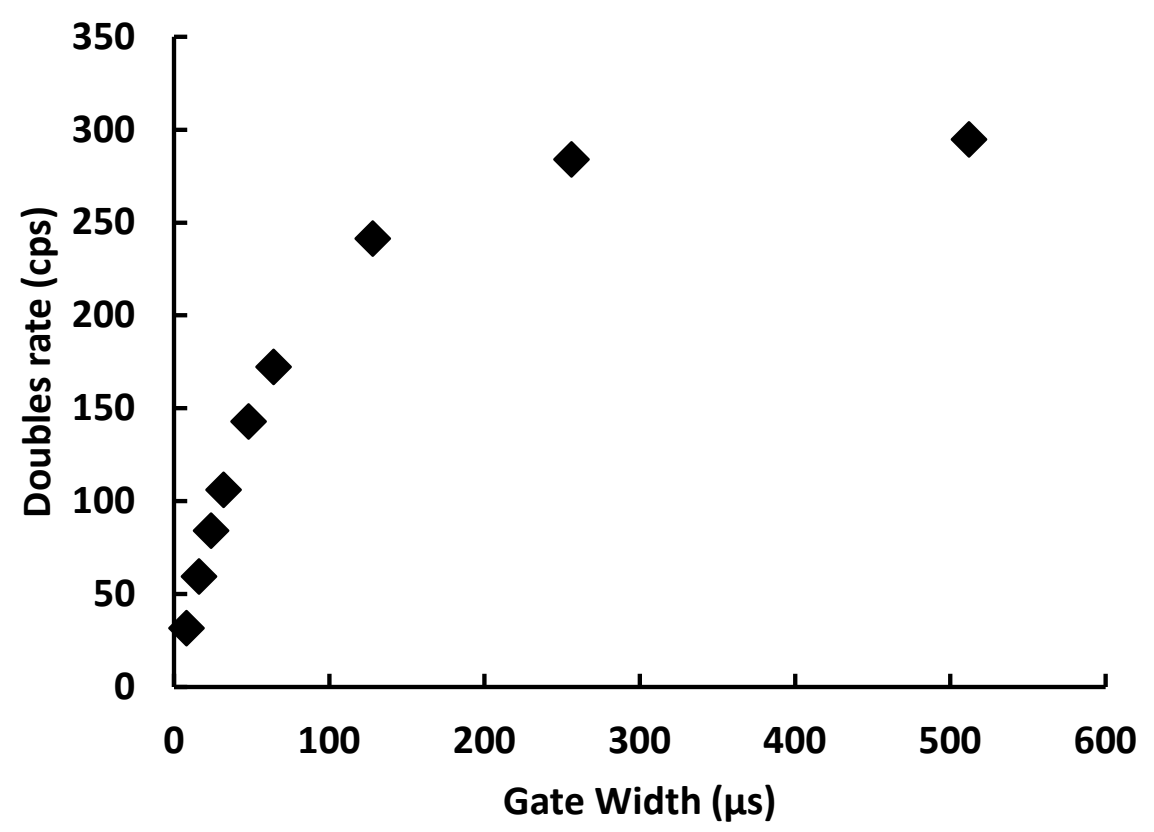

Figure 9. Simulated thermal mode doubles rate as a function of coincidence gate width for a 1,000 second count time.

Figure 10 shows the percentage relative uncertainty in the simulated thermal (no Cd) doubles neutron counting rate as a function of coincidence gate width for a 1,000 second count time. The relative uncertainty reaches a minimum at the point the maximum number of real coincidences are sampled from the neutron pulse train. However, as the coincidence gate width increases, more accidental coincidences are sampled and thus the relative uncertainty in the real coincidences or doubles rate reverses and begins to increase. 


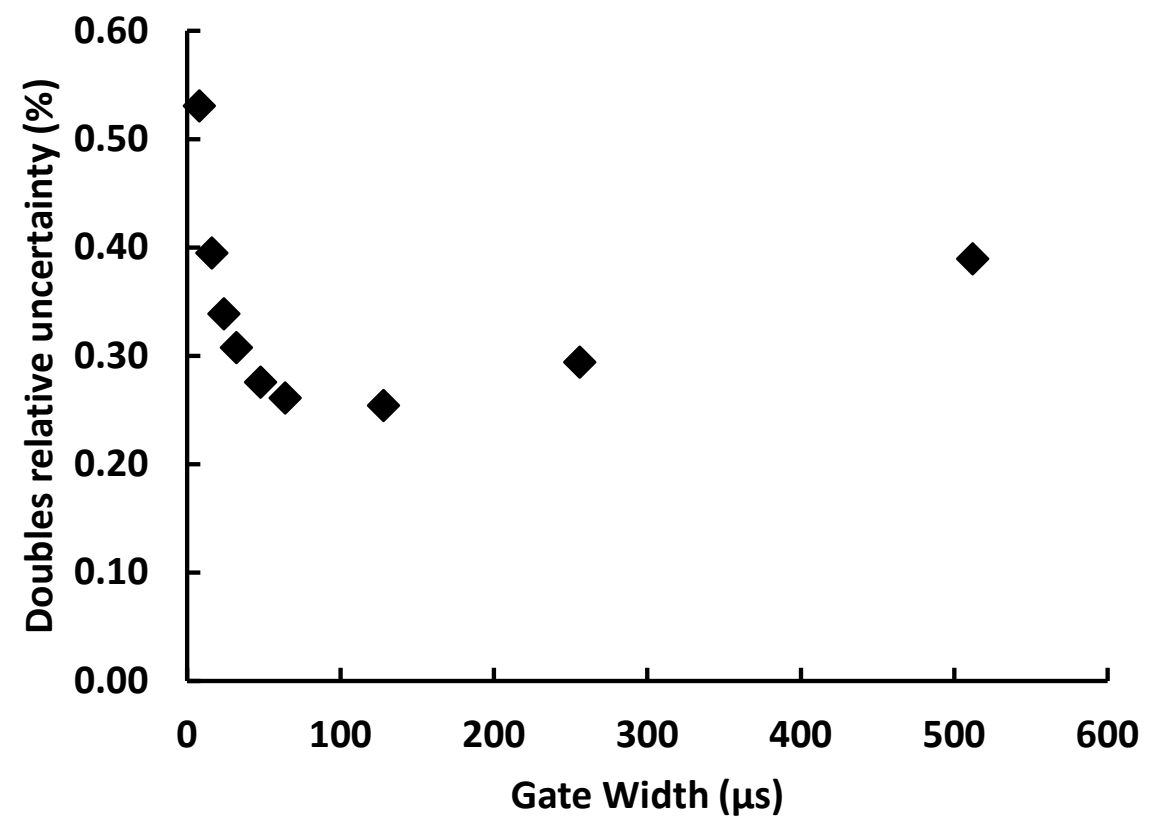

Figure 10. Simulated thermal mode doubles rate relative uncertainty as a function of coincidence gate width for a 1,000 second count time.

The list mode modeled results are provided in Section 4.5. The simulated count rates from the list mode measurements modeled results are produced from the same model as the typical measurements mode results. Therefore, the two sets of results were compared in terms of the relative neutron counting rates. For the 3,600 second assay of a full (intact) fresh fuel assembly with $4.5 \mathrm{wt} \%{ }^{235} \mathrm{U}$ enrichment with ${ }^{241} \mathrm{AmLi}(\alpha, \mathrm{n})$ neutron source strength of $5 \times 10^{4}$ neutrons s${ }^{-1}$, the thermal mode (no Cd) doubles neutron counting rate with $64 \mu$ s gate width was $172.3 \pm 0.9 \mathrm{~s}^{-1}(0.53 \%$ relative uncertainty $)$ across all detector channels. The total (singles) neutron counts detected across all detector channels was $(1.0616 \pm 0.0003) \times$ $10^{7}$ counts $(0.03 \%$ relative uncertainty). In the same mode, under the same conditions and counting time, the list mode neutron counting rates per detector channel varied between a minimum total neutron count of $(3.261 \pm 0.006) \times 10^{5}$ counts $(0.2 \%$ relative uncertainty $)$ in detector channels 6 and 13 , and a maximum total neutron count of $(9.440 \pm 0.010) \times 10^{5}$ counts $(0.1 \%$ relative uncertainty) in detector channels 1 and 18. The doubles neutron count rate varied between a minimum of $0.29 \pm 0.04 \mathrm{~s}^{-1}(12.2 \%$ relative uncertainty) in detector channels 6 and 13, and a maximum doubles neutron count rate of $1.27 \pm 0.08 \mathrm{~s}^{-1}$ (6.2\% relative uncertainty). For the successful future adoption of any list mode analysis method, the trade-off between the neutron counting time and the statistics of the individual or combinatorial counting rates will need to be investigated.

\subsection{MODEL PHYSICS}

The simulations were run using the default physics in the received MCNP model. A comparison was performed using the new physics card specified in the MCNP input file: the FMULT card (Lawrence Livermore National Laboratory model) and the default physics originally used. This comparison showed that the use of the model physics increased the number of coincidence events (doubles) observed in each of the three detector banks per AmLi source particle. An overall increase was observed in the number of coincidence events (doubles) in all 18 detector channels when using the Lawrence Livermore National Laboratory model compared to the default MCNP physics. However, only the correlated events from fission are affected using the new physics card. As expected, this does not influence the singles rate. For future work, calculations could be repeated using MCNP-PoliMi for comparison. 


\section{LIST MODE RESPONSE MATRIX ANALYSIS CONCEPT}

Two primary avenues of research were pursued for advanced analysis algorithm development: (1) initial development of a list mode response matrix concept for visualization of the collected neutron data for change detection between assayed items (e.g., a ${ }^{252} \mathrm{Cf}$ source at different positions within a neutron collar; an intact fuel assembly compared with an assembly with missing fuel rods); and (2) initial development of data analysis algorithms using classification methods based on list mode data generated using radiation transport models of the neutron collar. This section summarizes the first effort, and the second is published in a separate report [1].

\subsection{PHYSICAL BASIS}

The physical basis of the list mode response matrix method is defined here. The geometric dependences of the neutron interaction processes within the neutron coincidence collar detection system are described, which reveal spatial information about the assay item. The way this analysis method will use list mode data to obtain this spatial information is then demonstrated using simulation results.

Detection events in the neutron coincidence collar detector originate from two primary neutron sources:

1. Neutrons emitted from the ${ }^{241} \mathrm{AmLi}(\alpha, \mathrm{n})$ interrogation source

2. Neutrons born from ${ }^{235} \mathrm{U}$ induced fission in the fuel

The detected singles (or total) neutron count rate is sensitive to all sources of neutrons but is dominated by the contribution from the AmLi interrogation source neutrons. AmLi source neutrons dominate the singles rate because they are emitted as single neutrons with a random time signature characteristic of the

${ }^{241} \mathrm{Am} \alpha$-decay, so there is no real correlation. On the other hand, the detected doubles (or real coincidence) neutron count rate is more sensitive to correlated neutrons from fission. Fission neutrons are emitted in groups or "bursts" that are highly correlated in time (emitted within $\sim 10^{-12} \mathrm{~s}$ of the initiating fission event). Therefore, the doubles rate provides a measure of the ${ }^{235} \mathrm{U}$ induced fission rate in the fresh fuel assembly and thus the ${ }^{235} \mathrm{U}$ fissile mass [12].

The ability to use the individual count rates to distinguish between the time signature for AmLi source neutron and the time signature for induced fission neutrons forms the basis of the neutron coincidence method for fresh fuel verification measurements. The difference in the magnitude of the contribution of both AmLi and induced fission neutrons to the individual count rates forms the basis of the coincidence method for fresh fuel verification. Correlated neutron or coincidence counting is not in itself a new concept. However, detailed spatial effects within the collar have not previously been investigated and can be observed by using a full list mode approach. A detailed investigation of the spatial effects within the collar and how to leverage those for partial defect detection or nuclear design verification is new. Certain detectors or groups of detectors are more sensitive to either the AmLi source neutrons or the fission rate from individual fuel rods. This detection efficiency profile forms the basis of the list mode response matrix method. First, the geometry dependence of the neutron coincidence collar detection system is observed.

Figure 11 shows a two-dimensional (2D) histogram of neutron interactions in each fuel rod or fuel cell (fuel assembly within central box) as well as the histogram of neutron detections in each ${ }^{3} \mathrm{He}$ detector (circles around the box) labeled 1-18. This 2D histogram was generated using MCNP particle tracking (PTRAC) output file data. Neutron interactions - either fission events or detection events via captures in the ${ }^{3} \mathrm{He}$ gas - were tallied and plotted on a color scale. The color scale uses red or "hot" to represent the highest number of neutron interactions (fissions or detections via captures in the ${ }^{3} \mathrm{He}$ gas) and blue or "cold" to represent the lowest number of neutron interactions. The darkest blue squares represent the 
guide tubes and central instrument tube within the fuel assembly. Figure 11 shows that most induced fission events occur closest to the AmLi source, and the induced fission rate decreases throughout the fuel assembly as the fuel rods get further away from the AmLi source; consequently, the color map in the fuel changes from red to blue with a decreasing number of fissions. Figure 11 also shows that the anisotropic behavior affects the number of detected neutron events in the ${ }^{3} \mathrm{He}$ tubes. The detectors in the corners and furthest away from the AmLi neutron source (detectors 6,7,12, and 13) have the lowest number of detected neutrons (total or single neutron counts). This is also due to the corners of the collar that contain no polyethylene, which leads to a higher probability of neutron escape. Therefore, the number of neutrons thermalized and captured by the detectors near the corners is reduced relative to other detector locations. Detectors closest to the AmLi source have the highest number of detected neutrons because the probability of thermal neutron induced fission is highest closest to the source. Additionally, accidental coincidences may be observed from the AmLi source neutrons.

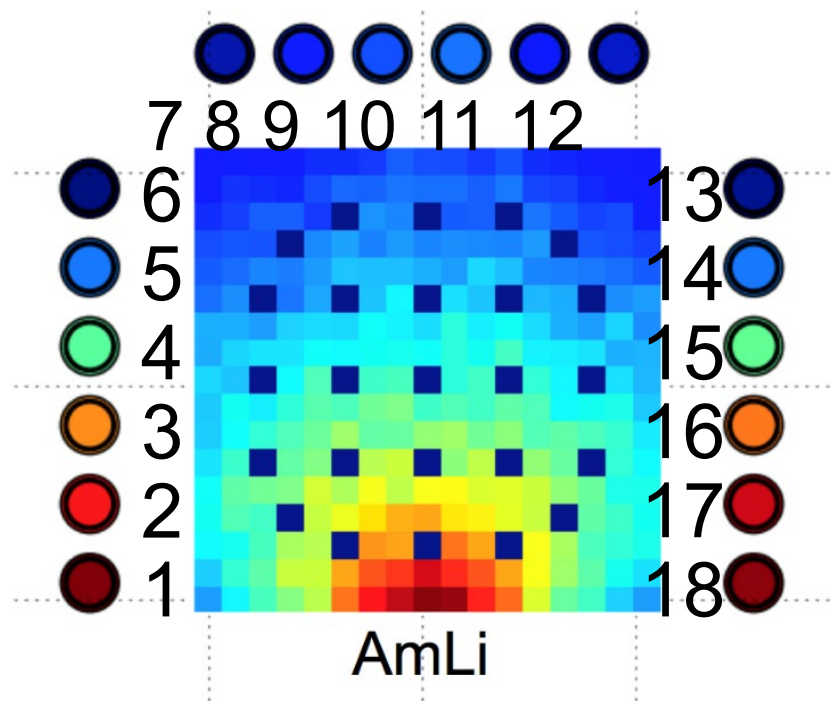

Figure 11. 2D histogram of neutron interactions in each fuel cell plus the histogram of neutron detections in each ${ }^{3} \mathrm{He}$ detector. Detectors are labelled 1-18.

MCNP was then used to understand the contribution of the two main neutron sources, AmLi and induced fission (i.e., the interrogating source signal or "background" and the fission signal of interest, respectively, to the singles neutron count rate). The MCNP PTRAC feature tracks neutrons from creation until loss including neutron captures on ${ }^{3} \mathrm{He}$. Therefore, the PTRAC output file enables each detection event (captures in ${ }^{3} \mathrm{He}$ ) to be traced back to one of these two neutron sources such that AmLi and fission neutrons can be distinguished using simulation. In turn, this provides an understanding of the physics of the features observed in the detected spatial response, which manifest themselves in the list mode response matrix. The MCNP PTRAC output file enables the origin of detected neutrons to be distinguished between ${ }^{241} \mathrm{AmLi}(\alpha, \mathrm{n})$ events from the interrogation source and induced fission events in the fuel. Since the signature of interest is the correlated neutron emission from induced fission, the list mode response matrix analysis method must be optimized to extract the most meaningful signature. The MCNP PTRAC output file facilitates this optimization.

Figure 12 shows the same behavior observed in Figure 11 but with higher fidelity. The bottom left of Figure 12 shows the individual contributions of induced fission in ${ }^{235} \mathrm{U}$ and the AmLi interrogating neutron source to the total number of neutron detections in each ${ }^{3} \mathrm{He}$ tube (for detectors $1-18$ ). The singles rate from the AmLi neutron source is much larger than that from ${ }^{235} \mathrm{U}$ fission, especially in the detectors closest to the AmLi neutron source, whereas the bottom right of Figure 11 shows the detectors in the outer corners have a higher fraction of single neutron detection events from ${ }^{235} \mathrm{U}$ induced fission. 

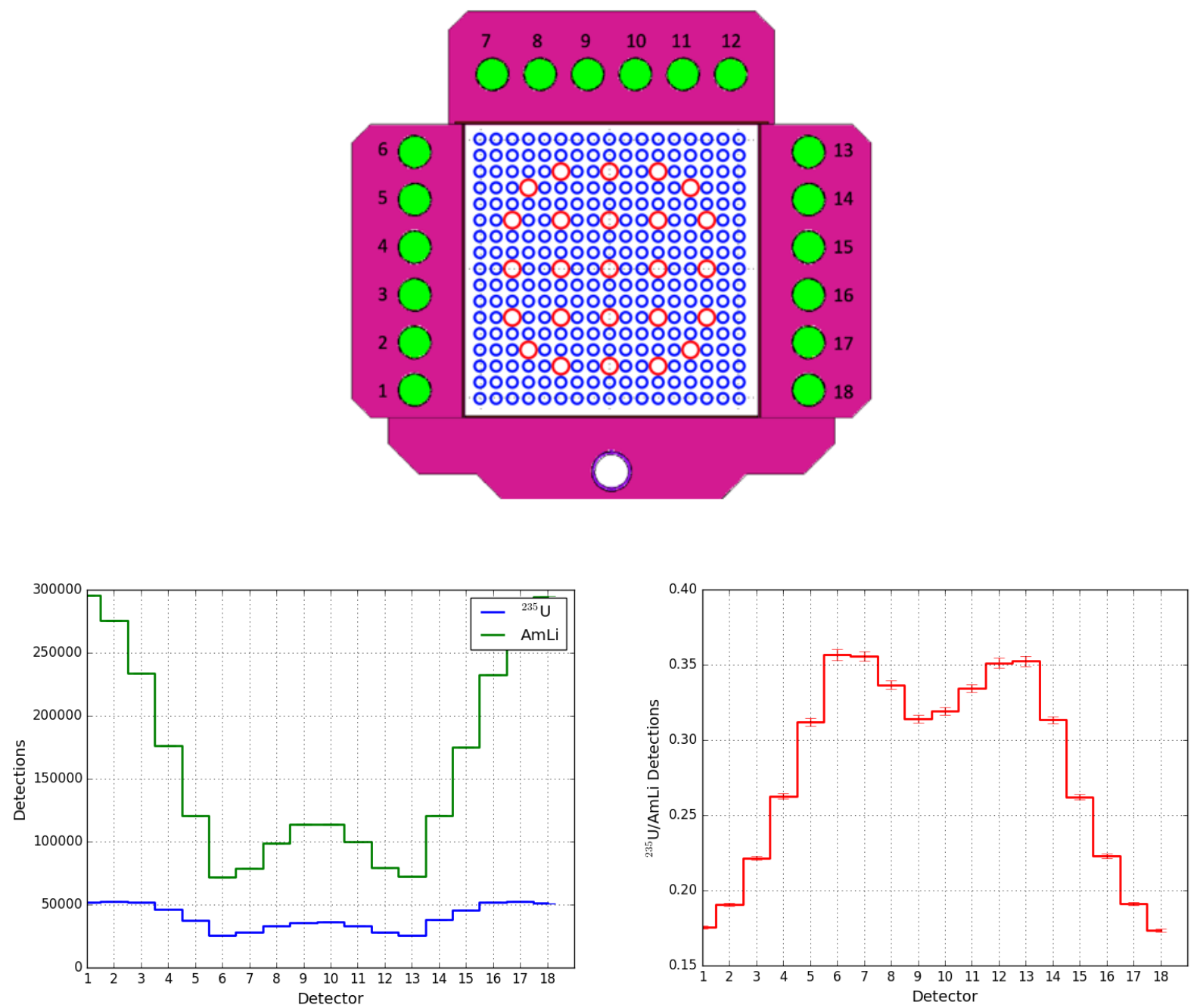

Figure 12. Simulated $17 \times 17$ full fresh fuel assembly within the neutron collar detector (top). Singles neutron counts in each detector from ${ }^{235} \mathrm{U}$ in fuel (blue) and AmLi (green) for a $5 \times 10^{4} \mathrm{n} / \mathrm{s}$ AmLi source over a 1 hour measurement time (bottom left). The ratio of ${ }^{235} \mathrm{U} / \mathrm{AmLi}$ singles neutron counts (Bottom right).

The neutron collar detector and fresh fuel assembly is a coupled system, and the detected response is highly dependent on the problem geometry. The list mode response matrix method under development leverages this geometric dependence of the detector response. This method provides an item-specific pattern of the detector spatial response that can be correlated to - and thus reveal - spatial information about the assay item. The neutron collar detector can be operated with and without a removable cadmium liner within the detector cavity in the fast and thermal modes, respectively. The liner modifies the interrogating spectrum and the spatial response function.

The method displays total counts and combinations of counts in the detectors, as well as correlations across channels, to provide a spatial indication of the presence of fissile material from the spatial response to induced fission and the corresponding absence of induced fission by the change in the spatial response. The term matrix captures the recording of counts with the diagonal elements of the matrix being the counts detected in an individual channel and the off-diagonal elements corresponding to correlations in multiple detector channels. At present, the matrix uses only correlated pairs and is $2 \mathrm{D}$. In principle, extending the method to include higher-order correlations and more dimensions is possible. The thinking 
behind the development of a matrix to display the spatial response is that it will form a recognizable "pattern" that can be correlated to the assay item. The method does not provide an "image" per se because the pattern is that of the detectors and detected response and not the fuel rods themselves.

A neutron imaging type of analysis approach has been explored for individual neutron slab detectors and total neutron counting [13]. However, this is the first time that this has been applied to safeguards correlated neutron counting using a neutron coincidence collar.

\subsection{MATRIX CONSTRUCTION}

Figure 13 shows the first construction of the list mode response matrix of a simulated full (i.e., complete and without gross or partial defects) fresh fuel assembly. This matrix is a $2 \mathrm{D}$ histogram of multiple neutron detections made for an AmLi source of strength $5 \times 10^{4} \mathrm{n} / \mathrm{s}$ over a 1 hour measurement time. The matrix indices [i,j] are $18 \times 18$ to correspond to the 18 detector channels. The diagonal matrix elements $([1,1],[2,2],[3,3]$, etc.) show multiple neutron events (i.e., neutrons from a chain induced by an AmLi neutron) detected in the same detector. In this case, the matrix elements represent pairs of detected events. The off-diagonal matrix elements show neutrons from the same fission event that are detected in both detectors (e.g., $[1,2]$ means that correlated neutrons were detected in detectors 1 and 2). More coincidences occur for detectors closest to the AmLi source (detectors 1,2,3,16,17, and 18), which is due to the fission rate being highest closest to the source.

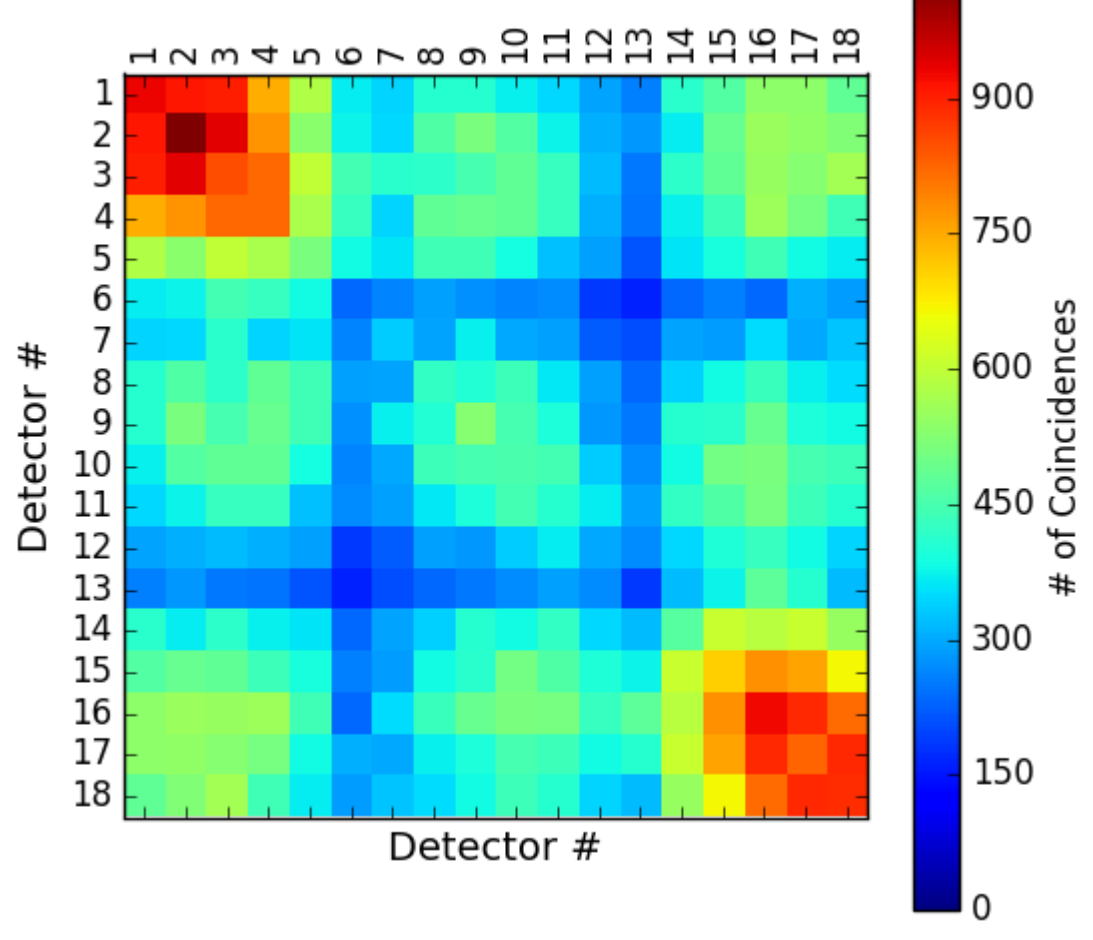

Figure 13. 2D histogram of multiple detections for an AmLi interrogation source of strength $5 \times 10^{4}$ neutrons $\mathrm{s}^{-1}$ over 1-hr measurement time in response to a full fresh fuel assembly.

This initial evaluation shows that the detected response reveals spatial information about the problem geometry in the case of the full fresh fuel assembly. Fuel rods closest to the AmLi source produce more fission events, which are detected more often by nearby detectors. This explains the "hot" regions around detectors $1-4$ and $15-18$. The asymmetries in the corners (e.g., between $[2,2]$ and $[17,17])$ are due to 
counting statistics. Detectors 6,7 and 12, 13 are in the outer corners, where induced fission in nearby fuel rods is small.

\subsection{CHANGE DETECTION}

The next step in the development of the list mode response matrix was to determine its sensitivity to spatial change. Both gross and partial defect scenarios were modeled using MCNP and the detected neutron count rates were simulated. The chosen gross defect scenario was to remove 66 pins $(\sim 25 \%)$ of the fresh fuel assembly. Figure 14 shows the list mode response matrix response for a fresh fuel assembly with the bottom left hand quadrant (per the plan view in the MCNP model) removed. The difference between Figure 14 and the bottom right of Figure 15 shows that the number of coincidences in the detector pairs closest to the bottom left hand corner of the fresh fuel assembly decreases.
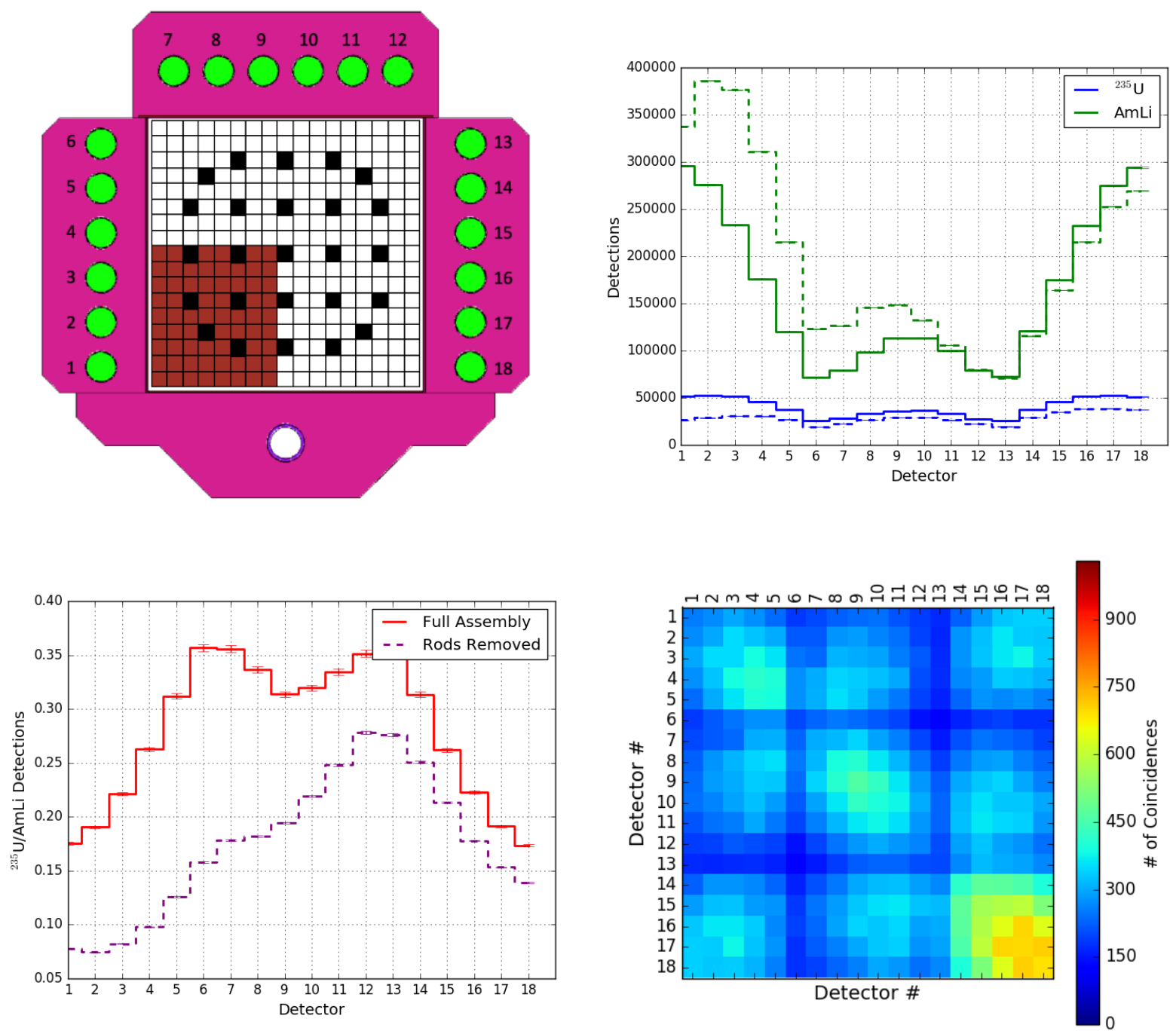

Figure 14. (Top left) UNCL detector model. The brown portion of the fuel assembly is missing. (Top right) Singles neutron counts in each detector from ${ }^{235} \mathrm{U}$ in fuel (blue) and AmLi (green) for a $5 \times 10^{4} \mathrm{n} / \mathrm{s} \mathrm{AmLi} \mathrm{source}$ over a 1 hour measurement time. (Bottom left) The ratio of ${ }^{235} \mathrm{U}$ to AmLi singles rates for the full assembly and particle defect. (Bottom right) 2D histogram of multiple neutron detections. 
Figure 15 shows the effect more clearly. The top left of the figure shows the $2 \mathrm{D}$ histogram from Figure 13 for a full fresh fuel assembly; the top right of the figure shows the 2D histogram from Figure 14 with 66 fuel rods removed from the bottom corner of the fuel assembly; and the bottom of the figure shows the difference between these two spatial responses. The difference histogram clearly indicates the removal of fuel in the region closest to detectors 1,2 , and 3 , which is the correct region. 

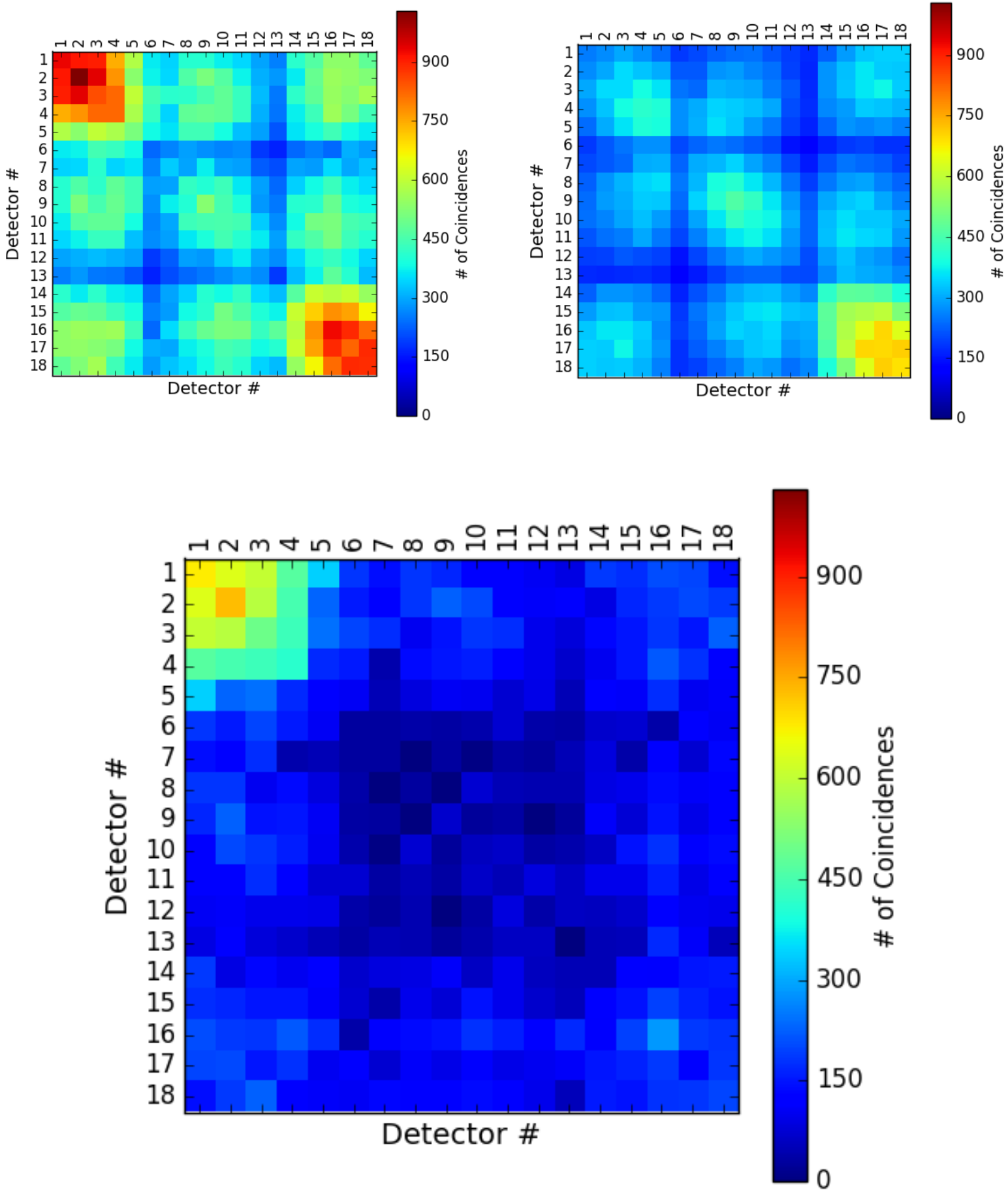

Figure 15. 2D histogram of multiple neutron detections for a $5 \times 10^{4} \mathrm{n} / \mathrm{s} \mathrm{AmLi} \mathrm{source} \mathrm{over} \mathrm{a} \mathrm{1-hr}$ measurement time for a full assembly (Top Left) and 66 rods removed (Top Right), and the difference (Bottom Center) between full and partial defect scenario.

Future research would include using analysis methods to trace the count rates back to the fuel pin of origin and generate an efficiency map. The starting point for future analysis is discussed in Section 4.4. 
Another research question for future exploration is whether there are assay scenarios where the gross material, but the list mode response matrix would reveal a difference.

\subsection{ALTERNATIVE APPROACH USING NEUTRON COINCIDENCE IMAGING}

In a neutron coincidence counting experiment, the sample fissile linear mass density $(m)$ is proportional to the neutron coincidence (or doubles) rate $d$ :

$$
d=c \varepsilon m
$$

where $c$ is a calibration constant and $\varepsilon$ is the efficiency matrix of a fuel rod producing coincidence neutrons in a detector. In the equation above, $d$ may be a vector of a set of detector networks, and $m$ may be a vector of all fuel rod linear mass density. The efficiency matrix is not necessarily square and is calculated in Monte Carlo simulations using a PTRAC analysis. In this analysis the efficiency matrix size is $n \times l$, where $n$ is the number of detector networks and $l$ is the number of fuel rods.

The PTRAC analysis is performed as follows. For each AmLi source history, neutron captures in each detector are recorded, including the detector index, fuel rod location, and time of the fission. These data are then processed to build the efficiency matrix. For each AmLi source history, if more than one neutron was detected in a detector network, the efficiency matrix element corresponding to that network and the fuel rod location is updated. The calculated efficiency matrix for a full fuel assembly is shown in Figure 16.
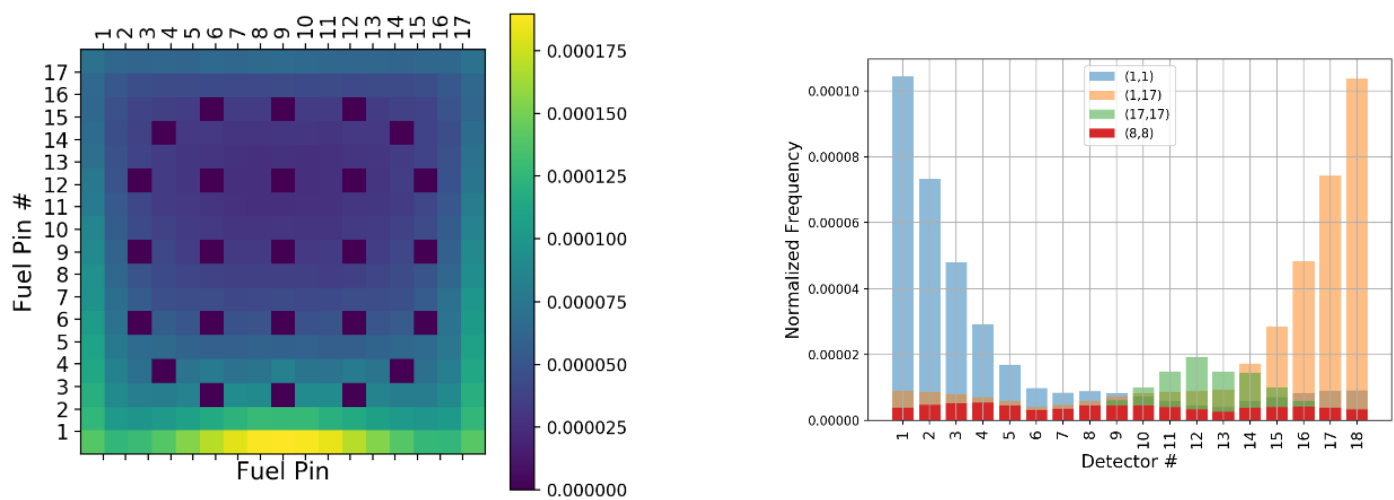

Figure 16. (Left) Pair efficiency matrix summed over all detectors for each fuel rod. (Right) Efficiency of captures in each detector for four individual fuel rods.

The left of Figure 16 shows the pair efficiency matrix summed over all detectors for each fuel rod within the $17 \times 17$ fuel rod array. The number of neutron captures per AmLi source history and thus neutroninduced fissions occurring in the fuel is highest at the front of the assembly closest to the source. Therefore, the calculated efficiency matrix is higher at those rod locations closest to the source (shaded yellow). The right of Figure 16 shows the efficiency of captures in each detector for four individual fuel rods at matrix locations $(1,1),(1,17),(17,17)$ and $(8,8)$. Locations $(1,1)$ and $(1,17)$ are the front left and right fuel rod locations, respectively, thus the efficiency of captures is highest in the detectors closest to those fuel rods (i.e., detectors 1 and 18). Then, this efficiency decreases as the detectors are farther away from those fuel rod locations. Fuel rod location $(8,8)$ is close to the center of the fuel rod array and, therefore, close to the center of the neutron collar detector array and approximately equidistant from all 
neutron detectors. Therefore, the efficiency of captures in each detector for rod $(8,8)$ is approximately constant across all detectors. Lastly, rod $(17,17)$ is in the top right corner of the fuel rod array and closest to detector 18. Therefore, this rod yields a maximum neutron capture efficiency in detector 18 and essentially mirrors the trend seen for rod $(1,1)$. These results demonstrate the rich amount of spatial information that can be obtained from this type of list mode analysis.

After an experiment, the doubles rate, calibration constant, and efficiency matrix are known, but the linear mass density is not. The fuel rod mass is solved using iterative methods such as nonnegative least squares or maximum likelihood algorithms. The normalized linear mass solution for a full fuel assembly is shown in Figure 17.

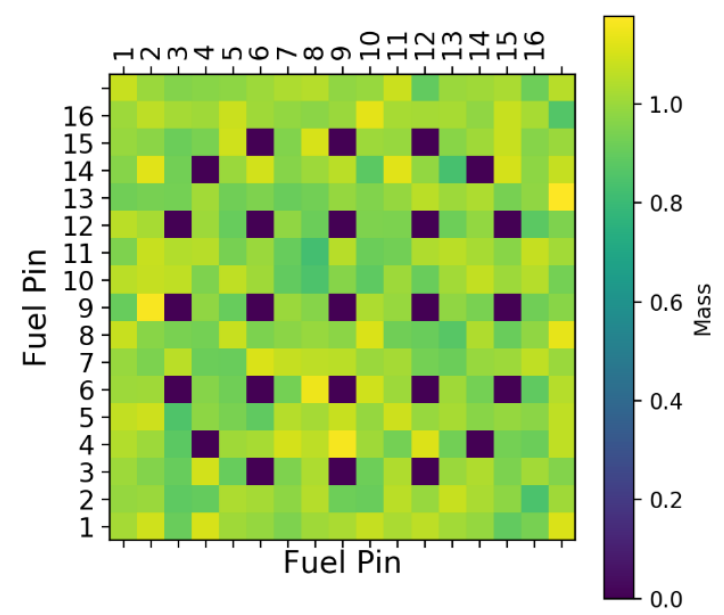

Figure 17. Calculated linear mass density for a full fuel assembly using a least squares algorithm on MCNP PTRAC calculated doubles rate and mass efficiency matrix.

These preliminary results indicate the potential of this method for future exploration. It was concluded that classification algorithms had greater potential for the list mode collar analysis algorithms, which was the chosen development path presented in a separate report [1]. However, the list mode method should be explored further in future research for other safeguards applications.

\subsection{PRELIMINARY STATISTICAL EVALUATION AND FEASIBILITY STUDY}

The 2D histograms are indicative of the ability of the method to detect gross spatial changes within the fresh fuel assembly, but they do not provide confidence levels at present. This analysis must not only detect change (gross spatial change), but also ensure confidence in the cause of the change outside normal conditions (e.g., counting statistics). Furthermore, this analysis must ensure that this can be done in a timely manner (i.e., at timescales commensurate with typical inspection times). A preliminary evaluation of the counting statistics of the method is shown below to begin to assign confidence intervals to the measurement. It is also important to consider the location of the missing rods relative to the AmLi source, and whether rods are removed in a cluster or distributed throughout the fuel assembly.

The response of the detector array to partial defects was also studied. The first step was to account for the time-dependence of the problem by postprocessing the MCNP output files. Figures 18 and 19 present simulated results for the variation in the total neutron count rate as a function of detection number for the removal of eight pins ( $3 \%$ of the assembly) from different locations within the fuel assembly. Results were simulated for a 1 hour count time with an AmLi source strength of $5 \times 10^{4}$ neutrons s ${ }^{-1}$ by postprocessing the MCNP file. These W-plots show the sensitivity of the total neutron counts to the 
position of the fuel pin removal. A change in the total neutron counts can be observed in detector locations 1-3 with the eight pins removed from the bottom corner of the assembly. When fuel is removed, it could be expected that the total neutron counts would decrease. However, an increase in the total number of counts can be observed in detectors close to the AmLi source because of the transmission of AmLi source neutrons through the empty pin locations. This trend reverses, and a signal decrease is observed for detectors farther away from the AmLi source. These results indicate that there might be benefit in taking ratios of detectors at the front and back of the collar. Furthermore, where the effect of fuel removal is canceled out in the singles rate (i.e., reduction in the count rate of the missing rods made up by streaming AmLi neutrons), the fuel removal would be revealed by the list mode matrix because it relies on doubles. These results were for clusters of pins and the results will be different for pin removal that is distributed throughout the assembly.

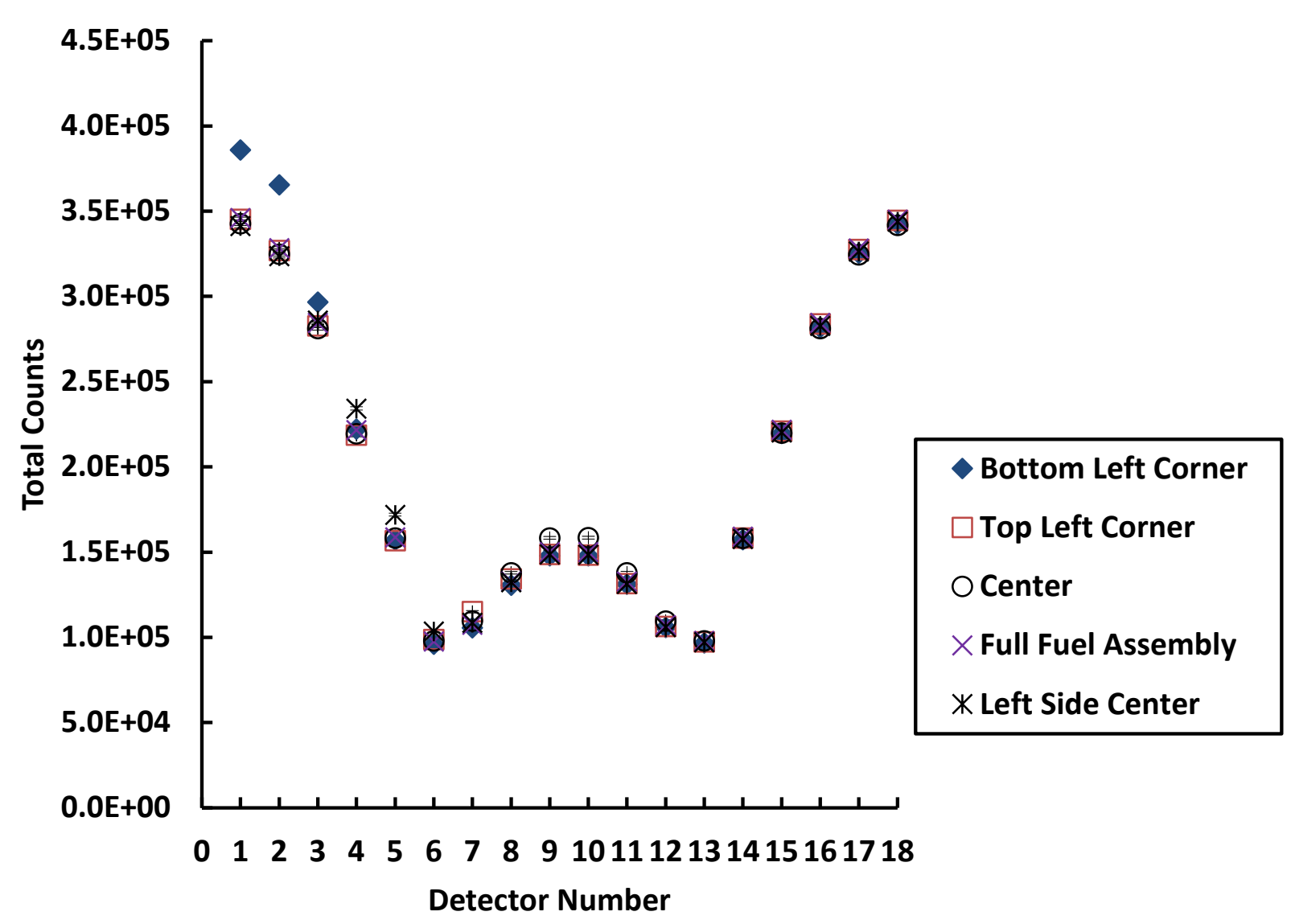

Figure 18. Singles or total neutron counts as a function of detector number in response to a full fuel assembly and a group of eight pins removed in different locations throughout the fuel assembly. This plot shows data for the neutron collar detector operated in the Cd ("fast") mode with a $5 \times 10^{4} \mathrm{n} / \mathrm{s} \mathrm{AmLi} \mathrm{source} \mathrm{for} \mathrm{a} 1$ hour measurement time. 


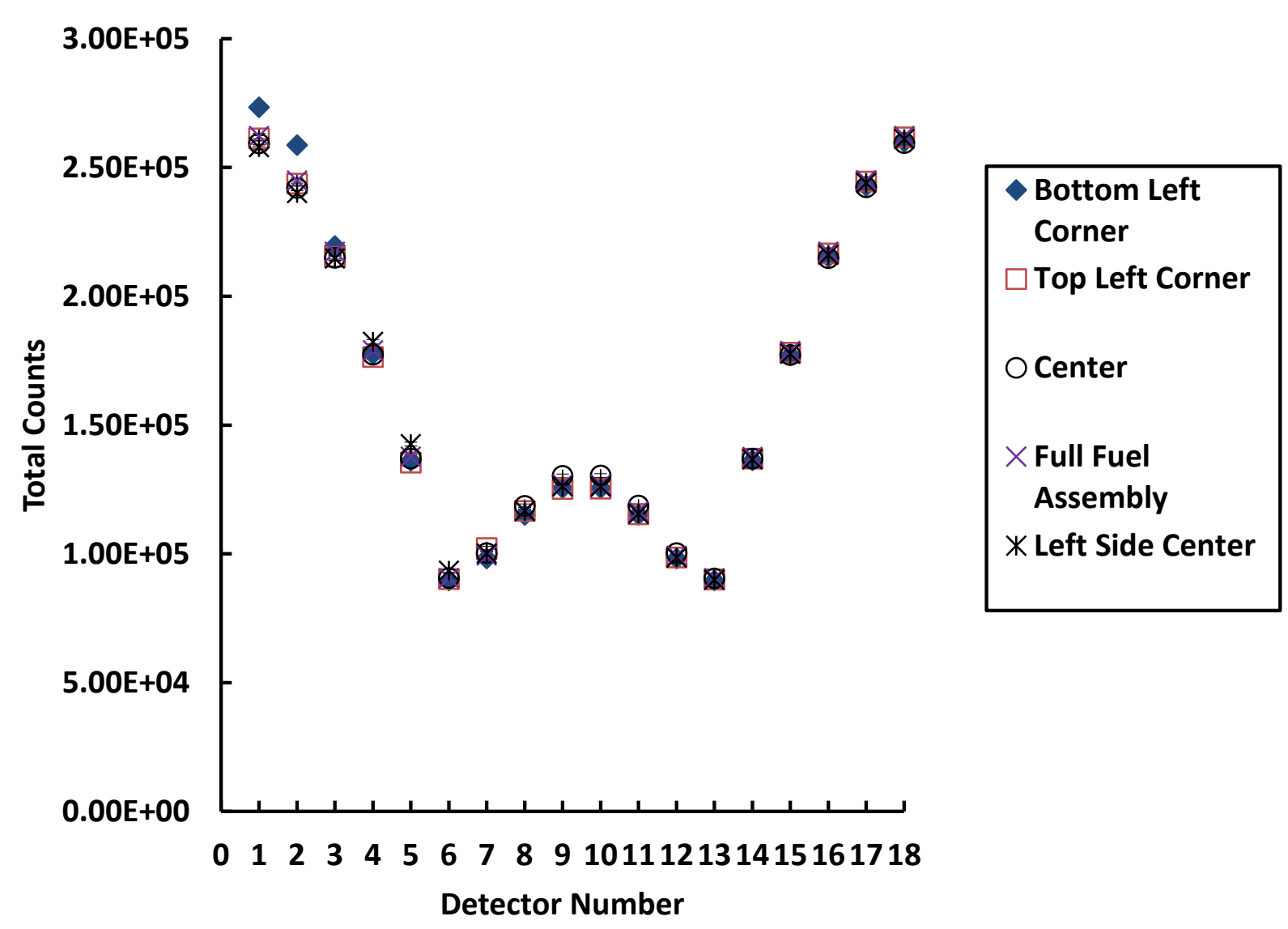

Figure 19. Singles or total neutron counts as a function of detector number in response to a full fuel assembly and a group of eight pins removed in different locations throughout the fuel assembly. This plot shows data for the neutron collar detector operated in the No-Cd ("thermal") mode with a $5 \times 104 \mathrm{n} / \mathrm{s}$ AmLi source for a 1 hour measurement time.

These preliminary results indicate that spatial changes can be detected outside counting statistics, but more work needs to be done to assign appropriate confidence intervals and generate confidence in the method. It is interesting to observe the same overall shape and trend as the measured data in Section 3. Furthermore, differences can be observed in the W-plot between the fast and thermal mode, which should be the subject of future exploration. For example, the neutron counting rates are higher in the fast mode toward the "edges" of the W-plot because the neutrons from the source have a greater probability of neutron capture in the fuel rods closest to the source and greater penetrability through the fuel assembly and thus a greater probability for inducing fission in the pins at the front perimeter of the assembly. Therefore, this shows that there might be a measurable difference in the fission rate between the two modes, particularly between the front and back of the assembly, that could add even more spatial information to this type of list mode analysis.

\section{SUMMARY}

This research provided a new LMCL neutron detection system, which was developed by a retrofit and modernization of an existing operational system, the JCC-71 neutron coincidence collar. The ability to resolve spatial information is a new capability realized by this retrofit. In addition, the electronics developed in this work can be retrofit to other existing neutron counting systems, including neutron 
multiplicity counters, as a cost-effective way to maintain and sustain the current fleet and simultaneously expand analysis capability. The retrofit successfully transitioned the novel technology from TRL-3 to TRL-5 over the course of the 3 year project.

This research also investigated through simulation the technical feasibility of using existing neutron infield tools in an innovative way to address the safeguards need for partial defect detection in fresh LEU fuel assemblies containing burnable poisons. Acknowledging limited IAEA resources, this work has investigated the potential to use existing neutron collar detectors leveraged in a new assay mode, the list mode response matrix, to alleviate the costs associated with designing, building, and testing a new instrument. The developed conceptual analysis method provides the technical foundation for later extension to the assay of more complex safeguards measurement scenarios including fresh mixed oxide fuel assemblies and fresh fuel assemblies in shipping containers. Furthermore, this analysis could be applied to new systems such as the IAEA fast neutron coincidence collar, which has 12 detectors. Three research avenues were explored for the development of analysis algorithms based on a list mode approach: (1) the list mode response matrix, (2) neutron coincidence imaging, and (3) classification algorithms. The latter was explored in greater detail and is published in a separate technical report [1], which is summarized here.

From Ref. [1], a relevance vector machine classifier (RVM-C) was developed to identify and localize partial defects within fresh fuel assemblies. A proof-of-concept data analysis was performed on a single simulated dataset comprised of one fuel assembly type and one partial defect scenario: (1) a $17 \times 17$ PWR fuel assembly in a normal configuration (i.e., all fuel rods present) and (2) one off-normal $25 \%$ fuel rod removal in a block (i.e., one quadrant of the fuel rods removed). This work demonstrated that list mode data may be analyzed using the RVM-C to differentiate normal fuel configurations from partial defects, and the position of the missing rods may also be identified. Pairwise coincidence rates were then analyzed to identify useful signatures for implementation of this classification method. In this approach, 71 detector networks were used to define coincident neutron detection events. In Ref. [1], a demonstration is provided of the variable-selection/basis-shaping routine integrated in the RVM-C training algorithm to rank the most useful of these detector networks for classifying the fuel defects.

\section{RECOMMENDATIONS FOR FUTURE WORK}

For future work, the following future modifications to the LMCL are recommended to further optimize the system:

- LVDS ribbon placement and security could be improved to avoid contact with the HV components including traces and components on the motherboard and the HV coupling capacitor.

- An improved version of the 3D-printed spacer using in each six-channel collar unit could be designed to better route the LVDS cables from each detector away from the nearby HV coupling capacitor and HV components/traces on the motherboard.

- Increasing the comparator hysteresis (set by a single resistor) should be considered to improve performance.

Further development is also needed to ensure that the new electronics are robust enough for transport and remain secure within the junction box.

Research activities that would benefit the future adoption of the project technology by potential end users include demonstration of the technology in a relevant operational environment using either a laboratory fresh fuel assembly or pin array (such as the fuel pin configuration at Los Alamos National Laboratory) or 
a real or representative fresh nuclear fuel assembly. List mode data should be acquired to test the algorithms and obtain a robust set of algorithms for future implementation in field-deployable software.

List mode analysis has potential application for verifying the burnable neutron absorber (poison) rod loading and configuration within fresh nuclear fuel assemblies. It could be used in the development of more accurate correction factors for that distribution [14]. Furthermore, there is the potential to apply the list mode approach to other neutron counting systems, especially benefitting those used for the assay of hard-to-measure and heterogeneous assay items including waste.

Future work is recommended to further develop analysis algorithms based on a list mode approach. These efforts should include work on the analysis algorithms, including simulating additional fuel configurations to test the ability of the developed list mode response matrix and RVM-C to identify and localize more subtle fuel defects. Variables we wish to explore include (1) fewer numbers of removed fuel rods (e.g., 1/8 assembly, eight rods, single rod), (2) removed rod position (e.g., back of assembly, front of assembly, center of assembly, edge of assembly), and (3) distribution of removed rods (i.e., a contiguous block versus randomly dispersed through the rod array) [1].

\section{REFERENCES}

1. Kenneth Dayman, Andrew Nicholson, Louise Worrall, Updates to the Relevance Vector Machine: Multiclass Classification, Variable Selection, and Proof-of-Concept Application to Safeguards Fresh Fuel Verification Using List-Mode Neutron Collar Data, Oak Ridge National Laboratory, ORNL/TM2018/1079, June 2020.

2. International Atomic Energy Agency, Symposium on International Safeguards: Building Future Safeguards Capabilities, International Atomic Energy Agency, November 5-8, 2018, STR-392, July 2019.

3. A. S. Moore, L. G. Worrall, C. Britton, S. Croft, K. Dayman, M. N. Ericson, R. D. McElroy, A. D. Nicholson, G. Nutter, R. B. Warmack, and J. P. Hayward, "Development and Evaluation of a List Mode Neutron Coincidence Collar for Spatial Response Measurements of Fresh Fuel Assemblies," Nuclear Instruments and Methods in Physics Research A, vol. 976, 2020.

4. Fuel design data, Nuclear Engineering International, September 2005.

5. International Atomic Energy Agency, "The Present Status of IAEA Safeguards on Nuclear Fuel Cycle Facilities," IAEA-Bulletin, vol. 22, no. 3/4.

6. Mirion Technologies (Canberra) Inc., JSR-15 ${ }^{\mathrm{TM}}$ Handheld Multiplicity Register (HHMR), Data Sheet, C0546-09/2017, 2017.

7. A. T. Simone, S. Croft, J. P. Hayward, and L. G. Worrall, "Using the JCC-71 Neutron Coincidence Collar as a Benchmark for Detector Characterization with PTR-32 List Mode Data Acquisition," Nuclear Instruments and Methods in Physics Research A, vol. 908, pp. 24-34, 2018.

8. L. G. Worrall, C. L. Britton Jr., S. Croft, K. Dayman, M. N. Ericson, R. D. McElroy Jr., A. D. Nicholson, A. T. Simone, Verification Data Pattern Recognition and Change Detection at the Neutron Instrument Level, Published in Proceedings of the IAEA Safeguards Symposium, Paper 292, Vienna, Austria, November 2018.

9. Canberra, "Model JCC-71, 72 and 73 Neutron Coincidence Collars," Fact Sheet C38898, 7/2011, 2011. 
10. T. Goorley, M. James, T. Booth, F. Brown, J. Bull, J. Cox, J. Durkee, J. Elson, M. Fensin, R. A. Forster, S. Mashnik, G. McKinney, D. Pelowitz, R. Prael, J. Sweezy, L. Waters, T. Wilcox, and T. Zukaitis, "Initial MCNP6 Release Overview," Nuclear Technology, vol. 180, pp. 298-315, 2012.

11. A. Belian et al., "Advanced Neutron Detection Technology Rodeo," 2017 ESARDA Symposium, Dusseldorf, Germany, 2017.

12. H. O. Menlove, Description and Performance Characteristics for the Neutron Coincidence Collar for the Verification of Reactor Fuel Assemblies, Los Alamos National Laboratory, LA-8939-MS (ISPO142), August 1981.

13. Robert J. Estep, Glen Brunson, and Sheila Melton, Neutron Coincidence Imaging for Active and Passive Neutron Assays, In Proceedings of the Institute of Nuclear Materials Management (INMM) Annual Meeting, July 2001.

14. L. G. Evans, M. T. Swinhoe, H. O. Menlove, P. Schwalbach, P. De Baere, and M. C. Browne, "A New Fast Collar for Safeguards Inspection Measurements of Fresh Low Enriched Uranium Fuel Assemblies Containing Burnable Poison Rods," Nuclear Instruments and Methods in Physics Research A, vol. 729, pp. 740-746, 2013. 
